Pure and Applied Mathematics Quarterly

Volume 4, Number 2

(Special Issue: In honor of

Fedor Bogomolov, Part 1 of 2$)$

$547-586,2008$

\title{
The Classification of Surfaces with $p_{g}=q=0$ Isogenous to a Product of Curves
}

\author{
I. C. Bauer, F. Catanese, F. Grunewald \\ Dedicated to Fedor Bogomolov on the occasion of his 60th birthday
}

\begin{abstract}
We classify all the surfaces with $p_{g}=q=0$ which admit an unramified covering isomorphic to a product of curves. Beyond the trivial case $\mathbb{P}^{1} \times \mathbb{P}^{1}$ we find 17 families which we explicitly describe. We reduce the problem to a combinatorial description of certain generating systems for finite groups which we solve using also MAGMA's library of groups of small order.
\end{abstract}

\section{Contents}

$\begin{array}{lll}0 . & \text { Introduction } & 549\end{array}$

1. Combinatorial preliminaries 554

1.1. Group theoretic terminology 554

1.2. Tuples 557

2. Basics on surfaces isogenous to a product 560

3. The unmixed case, classification of the groups 563

3.1. The case: $A=[2,3,7]_{84}, B \in \mathcal{N} \quad 565$

3.2. The case: $A=[2,3,8]_{48}, B \in \mathcal{N} \quad 568$

Received October 13, 2006. 
3.3. The case: $A, B \in \mathcal{N}_{3}, \alpha(A), \alpha(B) \leq 40 \quad 569$

3.4. The case: $A \in \mathcal{N}_{3}, \alpha(A) \neq 48,84, B \in \mathcal{N}_{4}, \mathcal{N}_{5}, \mathcal{N}_{6}$

3.5. The case: $A, B \in \mathcal{N}_{4}, \mathcal{N}_{5}, \mathcal{N}_{6} \quad 570$

4. The mixed case, classification of the groups 570

4.1. $A \in \mathcal{M}_{3}, \beta(A) \neq 16 \quad 571$

4.2. $A \in \mathcal{M}_{3}, \beta(A)=16 \quad 575$

4.3. $A \in \mathcal{M}_{4}, \mathcal{M}_{5}, \mathcal{M}_{6}, \mathcal{M}_{8} \quad 577$

5. Moduli spaces 578

6. Concrete models 581

6.1. $G=\mathfrak{A}_{5} \quad 581$

6.2. $G=\mathfrak{D}_{4} \times \mathrm{Z}_{2} \quad 581$

6.3. $G=\mathfrak{S}_{4} \quad 581$

6.4. $G=\mathfrak{S}_{4} \times \mathrm{Z}_{2} \quad 582$

6.5. $G=G(16) \quad 582$

6.6. $G=G(32), G(256,1), G(256,2) \quad 582$

References $\quad 585$ 


\section{INTRODUCTION}

It is well known that an algebraic curve of genus zero is isomorphic to the projective line. The search for an analogous statement in the case of algebraic surfaces led Max Noether to conjecture that a smooth regular (i.e., $q(S)=0$ ) algebraic surface with vanishing geometric genus $\left(p_{g}(S)=0\right)$ should be a rational surface. The first counterexample to this conjecture was provided by Federigo Enriques in 1896 ([Enr96], and also [EnrMS], I, page 294), who introduced the so called Enriques surfaces by considering the normalization of sextic surfaces in 3 -space double along the edges of a tetrahedron. Enriques surfaces are of special type, nowadays a large number of surfaces of general type with $p_{g}=q=0$ are known, but the first ones were constructed in the thirties by Luigi Campedelli and Lucien Godeaux (cf. [Cam32], [Go35]: in their honour minimal surfaces of general type with $K^{2}=1$ are called numerical Godeaux surfaces, and those with $K^{2}=2$ are called numerical Campedelli surfaces).

In the seventies, after rediscoveries of these old examples, many new ones were found through the efforts of several authors (cf. [BHPV], Vii, 10 and references therein). In particular, in the spirit of Godeaux' method to produce interesting surfaces as quotients $S=Z / G$ of simpler surfaces by the free action of a finite group $G$, Arnaud Beauville proposed a very simple construction by taking as $Z$ the product $Z=C_{1} \times C_{2}$ of two curves of respective genera $g_{1}:=g\left(C_{1}\right), g_{2}:=$ $g\left(C_{2}\right) \geq 2$, together with an action of a group $G$ of order $\left(g_{1}-1\right)\left(g_{2}-1\right)$ (this method produces surfaces with $\left.K^{2}=8\right)$. He also gave an explicit example as quotient of two Fermat curves (in [BaCa04] it was shown that his example leads to exactly two non isomorphic surfaces).

Generalising Beauville's construction we study here surfaces $S$ isogenous to a product of two curves, i.e., surfaces which have a finite unramified cover which is biholomorphic to a product of two curves. One says that the surface $S$ is isogenous to a higher product if both curves have genus bigger or equal to 2 (this condition is equivalent to $S$ being of general type).

It turns out that any surface with $p_{g}=q=0$ and isogenous to a product is either $\mathbb{P}^{1} \times \mathbb{P}^{1}$ or it is isogenous to a higher product (this happens since $\chi(S):=$ $\chi\left(\mathcal{O}_{S}\right)=1 \Longrightarrow \chi\left(C_{1} \times C_{2}\right)=\left(g_{1}-1\right)\left(g_{2}-1\right)>0$, whence either both $g_{i}$ 's are $\geq 2$, or both $g_{i}$ 's are $=0$ ). 
By results of [Cat00] any surface $S$ isogenous to a higher product has a unique minimal realization $S \cong\left(C_{1} \times C_{2}\right) / G$ where $G$ is a finite group acting freely on $C_{1} \times C_{2}$ and with $g_{1}:=g\left(C_{1}\right), g_{2}:=g\left(C_{2}\right) \geq 2$ chosen as small as possible. The action of $G$ can be seen to respect the product structure of $C_{1} \times C_{2}$. This means that there are the following two possibilities. Either there are actions of $G$ on $C_{1}$ and $C_{2}$ such that the action of $G$ on $C_{1} \times C_{2}$ is the diagonal action, and if this happens we speak of the unmixed case. Or there are elements in $G$ which interchange $C_{1}$ and $C_{2}$, and if this happens we speak of the mixed case. Obviously, in the mixed case $C_{1}$ and $C_{2}$ have to be biholomorphic to each other.

In this paper we carry out the classification of all smooth projective surfaces $S$ isogenous to a product with $p_{g}(S)=q(S)=0$. Note that if $S$ is of general type $p_{g}=0$ implies $q=0$, since for a surface of general type $\chi(S):=\chi\left(\mathcal{O}_{S}\right)=$ $1+p_{g}-q \geq 1$.

We can henceforth assume without loss of generality that $S \nsubseteq \mathbb{P}^{1} \times \mathbb{P}^{1}$ and therefore that $S$ is of general type.

First invariants of such surfaces are the group $G$ of the minimal realization $S \cong\left(C_{1} \times C_{2}\right) / G$ and the genera of $C_{1}$ and $C_{2}$.

It turns out that the surfaces $S$ which can be obtained for a fixed finite group $G$ and with fixed genera $g_{1}:=g\left(C_{1}\right), g_{2}:=g\left(C_{2}\right)$ fill out a finite number $N$ of irreducible connected components in the moduli space $\mathfrak{M}_{(1,8)}$ of minimal smooth complex projective surfaces with $\chi(S)=1$ and $K_{S}^{2}=8$. These turn out a posteriori to have the same dimension $D$.

Our main result is:

Theorem 0.1. If $S$ is a smooth projective surface isogenous to a product with $p_{g}(S)=q(S)=0$ and with minimal realization $S \cong\left(C_{1} \times C_{2}\right) / G$ then either $G$ is trivial and $S \cong \mathbb{P}^{1} \times \mathbb{P}^{1}$ or $G$ is one of the groups in the following table. The genera of the curves $C_{1}, C_{2}$ are as listed in the table. The number of the corresponding components $N$ in $\mathfrak{M}_{(1,8)}$ and their dimension are given in the remaining two columns. 


\begin{tabular}{|c|c|c|c|c|c|c|}
\hline$G$ & $|G|$ & Type & $g\left(C_{1}\right)$ & $g\left(C_{2}\right)$ & $N$ & $D$ \\
\hline $\mathfrak{A}_{5}$ & 60 & unmixed & 21 & 4 & 1 & 1 \\
$\mathfrak{A}_{5}$ & 60 & unmixed & 6 & 13 & 1 & 1 \\
$\mathfrak{A}_{5}$ & 60 & unmixed & 16 & 5 & 1 & 1 \\
$\mathfrak{S}_{4} \times \mathrm{Z}_{2}$ & 48 & unmixed & 25 & 3 & 1 & 3 \\
$\mathrm{G}(32)$ & 32 & unmixed & 5 & 9 & 1 & 2 \\
$\mathrm{Z}_{5}^{2}$ & 25 & unmixed & 6 & 6 & 2 & 0 \\
$\mathfrak{S}_{4}$ & 24 & unmixed & 13 & 3 & 1 & 3 \\
$\mathrm{G}_{(16)}$ & 16 & unmixed & 5 & 5 & 1 & 2 \\
$\mathfrak{D}_{4} \times \mathrm{Z}_{2}$ & 16 & unmixed & 9 & 3 & 1 & 4 \\
$\mathrm{Z}_{2}^{4}$ & 16 & unmixed & 5 & 5 & 1 & 4 \\
$\mathrm{Z}_{3}^{2}$ & 9 & unmixed & 4 & 4 & 1 & 2 \\
$\mathrm{Z}_{2}^{3}$ & 8 & unmixed & 5 & 3 & 1 & 5 \\
$\mathrm{G}(256,1)$ & 256 & mixed & 17 & 17 & 3 & 0 \\
$\mathrm{G}(256,2)$ & 256 & mixed & 17 & 17 & 1 & 0 \\
\hline
\end{tabular}

Here $\mathfrak{A}_{5}$ is the alternating group on 5 letters, $\mathfrak{S}_{4}$ is the symmetric group on 4 letters, $\mathfrak{D}_{4}$ is the dihedral group of order $8, \mathrm{Z}_{n}$ is the cyclic group of order $n, \mathrm{G}(32)$ and $\mathrm{G}(16)$ are two groups of respective orders 32,16 described in sections 3.5 and 6 and $\mathrm{G}(256,1), \mathrm{G}(256,2)$ are two groups of order 256 described in sections 4.2.2 and 6 .

We see our main result as the solution in a very special case to the open problem that David Mumford set forth at the Montreal Conference in 1980: "Can a computer classify all surfaces of general type with $p_{g}=0$ ? Our purpose is to show how computationally complex this question is and that probably computers are needed even if one asks a more restricted question.

All known surfaces of general type with $p_{g}=0, K^{2}=8$ are quotients $\mathbb{H} \times \mathbb{H} / \Gamma$ of the product of two upper half planes by a discrete cocompact subgroup of $\operatorname{PSL}(2, \mathbb{R}) \times \operatorname{PSL}(2, \mathbb{R})$. There are also quotients which are not related to products of curves; constructions of such surfaces using quaternion algebras have been known since long, see for example [Ku], [Sha78]. Also in this case a complete classification is possible. We shall elaborate on this in a forthcoming paper.

It is an interesting question whether there do exist surfaces of general type with $p_{g}=0, K^{2}=8$ which are not quotients $\mathbb{H} \times \mathbb{H} / \Gamma$ as above (observe that 
for $p_{g}=0, K^{2}=9$ the universal cover is the unit disk in $\mathbf{C}^{2}$ by Yau's theorem [Y77]). The question of existence of such surfaces of general type which are simply connected is attributed to Hirzebruch. Notice that if their intersection forms were even, they would be homeomorphic to $\mathbb{P}^{1} \times \mathbb{P}^{1}$ but not diffeomorphic, (see [Fre82] and [Don96]).

Surfaces with $p_{g}=q=0$ were also investigated from other points of view. We would like to mention several articles by M. Mendes Lopes and R. Pardini ([Pa03], [MLP01], [MLP03]) where the authors encountered them in the course of studying the failure of birationality of the bicanonical map.

Concrete examples of rigid surfaces isogenous to a product have been given in [BCG05].

Previously, in [BaCa04] the first two authors classified all smooth algebraic surfaces isogenous to a product and of unmixed type with $p_{g}=q=0$, and with $G$ a finite abelian group.

They also gave a complete description of the connected components of the moduli space that arise from these surfaces.

In this article we complete this classification admitting arbitrary groups and treating also the mixed type.

While describing the organisation of the paper we shall now explain the steps of our classification procedure in more detail.

A family of surfaces isogenous to a product with associated group $G$ and with $q=0$ is determined in the unmixed case by a set of data which we call a ramification structure. It consists of a pair of spherical systems of generators $\left[g_{(1,1)}, \ldots, g_{(1, r)}\right],\left[g_{(2,1)}, \ldots, g_{(2, s)}\right]$ for the group $G$ (i.e., a system of generators whose product equals the identity), which are 'disjoint' in the sense that the union of the conjugates of the cyclic subgroups generated by $g_{(1,1)}, \ldots, g_{(1, r)}$, resp. by $g_{(2,1)}, \ldots, g_{(2, s)}$ have trivial intersection. We exploit also the fact that the geometric conditions impose very strong restrictions on the possible orders of the elements $g_{(i, j)}$.

We are able to classify these by combinatorial methods of finite group theory. Riemann's existence theorem guarantees in fact that for any ramification structure there is an irreducible family of surfaces isogenous to a product with the given ramification structure. 
In the mixed case we follow a similar approach.

In section 1 we fix the algebraic set up and classify all the possible types (i.e., tuples of orders) of the spherical systems of generators. In fact, the conditions on the possible tuples of orders are strong enough to leave only finitely many possibilities also for the orders of the finite groups which have to be considered.

In section 1.1 we introduce the action of the product of a suitable braid group with $\operatorname{Aut}(G)$ on the set of 'disjoint' spherical systems of generators, which reflects the deformation equivalence of the associated surfaces.

In section 2 we collect some basic results on surfaces isogenous to a product and show how they correspond to the algebraic data introduced previously.

In sections 3, 4 we carry out the complete classification of all finite groups occuring as groups $G$ associated to a surface isogenous to a product with $p_{g}=0$. The procedure is simple: using the libraries of the MAGMA computer algebra system [MSG], which contain all groups of order less then 2000 (with the exception of 1024), we try to inspect all groups whose orders appear in the list obtained in section 1 asking for the existence of suitable systems of generators. This turns out not to be an easy task for two reasons: first, the number of groups which has to be checked is much too high to be feasible to a direct computer calculation, second, the orders of the groups in question may be too high to be contained in the standard group libraries. In order to prove our main result we have then to use direct arguments (exploiting for example the solvability of groups whose order admits only two prime factors), which allow us to reduce the cardinality of the finite groups under consideration until we reach a region which is covered by the MAGMA-library of small groups.

We believe that the interest of our paper is twofold: first of all the list of surfaces in Theorem 0.1 contains new and interesting examples. Finding them is difficult, but once their existence is established, they are easy to construct explicitly. Secondly, it seems interesting to us that it is possible to carry out a subcase of the Mumford classification program.

We did not try to eliminate as many computer calculations as possible, but we succeeded to keep the complexity and time requirements for each single calculation small. sections 3,4 are to be understood as a Leitfaden through a maze of little facts about finite groups. We would like to remark that some of our 
computer calculation could be replaced using the methods in [Breu] to show that under certain conditions a finite group cannot be a quotient of a given polyhedral group.

We devote section 6 to a simple description of the groups and ramification structures occurring. We hope that this description may be useful in order to carry out explicit calculations on our surfaces.

Finally, in section 5 we calculate the number of orbits of the direct product of suitable braid groups with $\operatorname{Aut}(G)$ acting on the set of disjoint pairs of spherical systems of generators. By this procedure we determine the exact structure of the corresponding subset of the moduli space corresponding to surfaces isogenous to a product with $p_{g}=0$ : in particular we determine the number of connected components (which are automatically irreducible) and their respective dimensions.

\section{Combinatorial Preliminaries}

This section contains simple combinatorial results which are important as a first step in the solution of the algebraic problem to which our classification can be reduced. We also fix certain terminologies to be used later. The reader who finds these preliminaries too dry to swallow might first want to read the subsequent section 2, explaining how we pass from geometry to algebra.

1.1. Group theoretic terminology. Let $G$ be a group and $r \in \mathbb{N}$ with $r \geq 2$. An $r$-tuple $T=\left[g_{1}, \ldots, g_{r}\right]$ of elements of $G$ is called a spherical system of generators of $G$ if $g_{1}, \ldots, g_{r}$ is a system of generators of $G$ (i.e., $G=\left\langle g_{1}, \ldots, g_{r}\right\rangle$ ) and we additionally have $g_{1} \cdot \ldots \cdot g_{r}=1$.

We call $r=: \ell(T)$ the length of $T$.

If $T=\left[g_{1}, \ldots, g_{r}\right]$ is an $r$-tuple of elements of $G$ and $g \in G$ we define $g T g^{-1}:=$ $\left[g g_{1} g^{-1}, \ldots, g g_{r} g^{-1}\right]$.

If $A=\left[m_{1}, \ldots, m_{r}\right] \in \mathbb{N}^{r}$ is an $r$-tuple of natural numbers with $2 \leq m_{1} \leq$ $\ldots \leq m_{r}$ then the spherical system of generators $T=\left[g_{1}, \ldots, g_{r}\right]$ is said to have type $A=\left[m_{1}, \ldots, m_{r}\right]$ if there is a permutation $\tau \in \mathfrak{S}_{r}$ such that

$$
\operatorname{ord}\left(g_{1}\right)=m_{\tau(1)}, \ldots, \operatorname{ord}\left(g_{r}\right)=m_{\tau(r)}
$$


holds. Here ord $(g)$ is the order of the element $g \in G$. The spherical system of generators $T=\left[g_{1}, \ldots, g_{r}\right]$ is said to be ordered if $2 \leq \operatorname{ord}\left(g_{1}\right) \leq \ldots \leq \operatorname{ord}\left(\mathrm{g}_{\mathrm{r}}\right)$.

Given a spherical system of generators $T=\left[g_{1}, \ldots, g_{r}\right]$ of $G$ we define its stabilizer set $\Sigma(T)$ as

$$
\Sigma(T)=\Sigma_{G}(T)=\Sigma\left(\left[g_{1}, \ldots, g_{r}\right]\right):=\bigcup_{g \in G} \bigcup_{j \in \mathbb{Z}} \bigcup_{i=1}^{r}\left\{g \cdot g_{i}^{j} \cdot g^{-1}\right\}
$$

to be the union of all conjugates of the cyclic subgroups generated by the elements $g_{1}, \ldots, g_{r}$. A pair of spherical systems of generators $\left(T_{1}, T_{2}\right)$ of $G$ is called disjoint if

$$
\Sigma\left(T_{1}\right) \cap \Sigma\left(T_{2}\right)=\{1\} .
$$

Definition 1.1. Consider an $r$-tuple $A_{1}=\left[m_{(1,1)}, \ldots, m_{(1, r)}\right]$ and a s-tuple $A_{2}=$ $\left[m_{(2,1)}, \ldots, m_{(2, s)}\right]$ of natural numbers with $2 \leq m_{(1,1)} \leq \ldots \leq m_{(1, r)}$ and $2 \leq$ $m_{(2,1)} \leq \ldots \leq m_{(2, s)}$. An unmixed ramification structure of type $\left(A_{1}, A_{2}\right)$ for $G$ is a disjoint pair $\left(T_{1}, T_{2}\right)$ of spherical systems of generators of $G$, such that $T_{1}$ has type $A_{1}$ and $T_{2}$ has type $A_{2}$. We define $\mathcal{B}\left(G ; A_{1}, A_{2}\right)$ to be the set of unmixed ramification structures of type $\left(A_{1}, A_{2}\right)$ for $G$.

Definition 1.2. Let $A=\left[m_{1}, \ldots, m_{r}\right]$ be an $r$-tuple of natural numbers with $2 \leq m_{1} \leq \ldots \leq m_{r}$. A mixed ramification structure of type $A$ for $G$ is a pair $(H, T)$ where $H$ is a subgroup of index 2 in $G$ and $T=\left[g_{1}, \ldots, g_{r}\right]$ is an $r$-tuple of elements of $G$ such that the following hold

- $T$ is a spherical system of generators of $H$ of type $A$,

- for every $g \in G \backslash H$, the $r$-tuples $T$ and $g T g^{-1}=\left[g g_{1} g^{-1}, \ldots, g g_{r} g^{-1}\right]$ are disjoint,

- for every $g \in G \backslash H$ we have $g^{2} \notin \Sigma_{H}(T)$.

We define $\mathcal{B}(G ; A)$ to be the set of mixed ramification structures of type $A$ for $G$.

We shall now establish certain equivalence relations on the sets $\mathcal{B}\left(G ; A_{1}, A_{2}\right)$ and $\mathcal{B}(G ; A)$ of ramification structures of a finite group $G$, which reflect the deformation equivalence of the surfaces admitting such ramification structures. This equivalence relation will be used in section 5 . 
Let $r$ be a natural number and consider the braid group

$$
\mathbf{B}_{r}:=\left\langle\begin{array}{c|c}
\sigma_{1}, \ldots, \sigma_{r-1} & \sigma_{i} \sigma_{j}=\sigma_{j} \sigma_{i} \text { if }|i-j|>1, \\
\sigma_{i} \sigma_{i+1} \sigma_{i}=\sigma_{i+1} \sigma_{i} \sigma_{i+1} \text { for } i=1, \ldots, r-2
\end{array}\right\rangle .
$$

Consider now the so-called Hurwitz action of $\mathbf{B}_{r}$ on the set of $r$-tuples of elements of $G$ corresponding to the standard embedding of $\mathbf{B}_{r}$ into the automorphism group of a free group on $r$ generators.

Let $T=\left[g_{1}, \ldots, g_{r}\right]$ be a $r$-tuple of elements of $G$ and $1 \leq i \leq r-1$. Define $\sigma_{i}(T)$ by

$$
\sigma_{i}(T):=\left[g_{1}, \ldots, g_{i-1}, g_{i} \cdot g_{i+1} \cdot g_{i}^{-1}, g_{i}, g_{i+2}, \ldots, g_{r}\right]
$$

It is well known and also easy to see that

i) the braid relations are satisfied,

ii) the group $\mathbf{B}_{r}$ maps spherical systems of generators to spherical systems of generators, preserving the type.

Also the automorphism group $\operatorname{Aut}(G)$ of $G$ acts on the set of spherical systems of generators of a fixed type by simultaneous application of an automorphism to the coordinates of a tuple.

Given $\left(\gamma_{1}, \gamma_{2}, \varphi\right) \in \mathbf{B}_{r} \times \mathbf{B}_{s} \times \operatorname{Aut}(G)$ and $\left(T_{1}, T_{2}\right) \in \mathcal{B}\left(G ; A_{1}, A_{2}\right)$, where $T_{1}$ has length $r$ and $T_{2}$ has length $s$, we set

$$
\left(\gamma_{1}, \gamma_{2}, \varphi\right) \cdot\left(T_{1}, T_{2}\right):=\left(\varphi\left(\gamma_{1}\left(T_{1}\right)\right), \varphi\left(\gamma_{2}\left(T_{2}\right)\right)\right) .
$$

A one moment's consideration shows that (4) leads to an action of $\mathbf{B}_{r} \times \mathbf{B}_{s} \times$ $\operatorname{Aut}(G)$ on $\mathcal{B}\left(G ; A_{1}, A_{2}\right)$.

Given $(\gamma, \varphi) \in \mathbf{B}_{r} \times \operatorname{Aut}(G)$ and $(H, T) \in \mathcal{B}(G ; A)$, where $T$ has length $r$, we set

$$
(\gamma, \varphi) \cdot(H, T):=(\varphi(H), \varphi(\gamma(T))) .
$$

Formula (5) leads to an action of $\mathbf{B}_{r} \times \operatorname{Aut}(G)$ on $\mathcal{B}(G ; A)$.

In section 2 we shall associate to a surface $S$ isogenous to a product of unmixed (resp.mixed) type with $q=0$ an equivalence class of an unmixed (resp. mixed) ramification structure for $G=G(S)$. In section 2 we shall also conversely see that an unmixed (resp. mixed) ramification structure for a finite group $G$ gives a surface $S$ isogenous to a product of unmixed (resp.mixed) type with $q=0$. The 
equivalence classes (the orbits of the respective actions) determine also exactly the irreducible components in the corresponding moduli space. This will be applied in section 5 .

In the following sections polygonal groups will play an important role. We give their definition right away. Let $A:=\left[m_{1}, \ldots, m_{r}\right]$ be a $r$-tuple of natural numbers $\geq 2$. The polygonal group $\mathbb{T}\left(m_{1}, \ldots, m_{r}\right)$ is defined by generators and relations as

$$
\mathbb{T}\left(m_{1}, \ldots, m_{r}\right):=\left\langle t_{1}, \ldots, t_{r} \quad \mid t_{1} t_{2} \ldots t_{r}=1=t_{1}^{m_{1}}=\ldots=t_{r}^{m_{r}}\right\rangle .
$$

These groups are important for us since every finite group which has a spherical system of generators of type $A$ is (in the obvious way) a quotient group of $\mathbb{T}\left(m_{1}, \ldots, m_{r}\right)$.

1.2. Tuples. In this section we classify $r$-tuples of natural numbers satisfying certain arithmetic conditions. The lists of these tuples will be of importance in our later classification program of surfaces.

For an $r$-tuple $(r \in \mathbb{N})\left[m_{1}, \ldots, m_{r}\right] \in \mathbb{N}^{r}$ define the orbifold canonical degree as

$$
\Theta\left(\left[m_{1}, \ldots, m_{r}\right]\right):=-2+\sum_{i=1}^{r}\left(1-\frac{1}{m_{i}}\right)
$$

In the following we define properties of tuples of natural numbers which are satisfied by the tuples of orders of the spherical systems of generators occurring in the unmixed case.

Definition 1.3. I) Given $A=\left[m_{1}, \ldots, m_{r}\right] \in \mathbb{N}^{r}$ define

$$
\alpha(A):=\frac{2}{\Theta(A)}=\frac{2}{-2+\sum_{i=1}^{r}\left(1-\frac{1}{m_{i}}\right)}
$$

and use the notation $\left[m_{1}, \ldots, m_{r}\right]_{\alpha(A)}$.

II) For $r \in \mathbb{N}$ with $r \geq 3$ let $\mathcal{N}_{r}$ be the set of $r$-tuples $\left[m_{1}, \ldots, m_{r}\right] \in \mathbb{N}^{r}$ which satisfy:

(i): $2 \leq m_{1} \leq \ldots \leq m_{r}$,

(ii): $\Theta\left(\left[m_{1}, \ldots, m_{r}\right]\right)>0$,

(iii): $\alpha\left(\left[m_{1}, \ldots, m_{r}\right]\right) \in \mathbb{N}$,

(iv): $m_{i}$ divides $\alpha\left(\left[m_{1}, \ldots, m_{r}\right]\right)$ for all $1 \leq i \leq r$. 
We set $\mathcal{N}:=\cup_{i=3}^{\infty} \mathcal{N}_{i}$.

We shall now give a simple classification result for the tuples in $\mathcal{N}$.

Proposition 1.4. We have $\mathcal{N}_{r}=\emptyset$ for $r \geq 7$ and

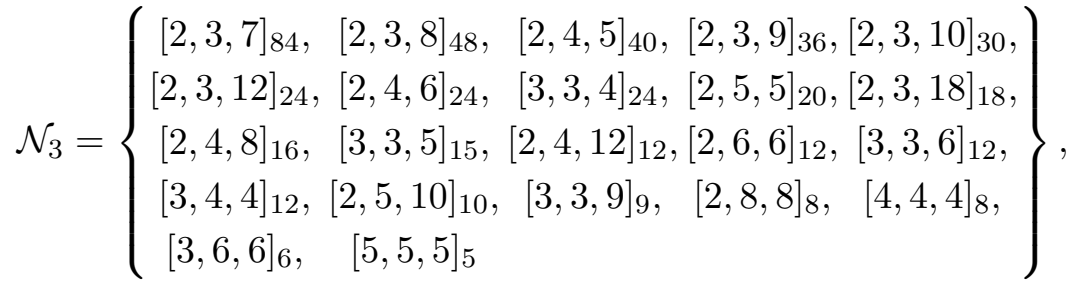

$$
\begin{aligned}
& \mathcal{N}_{4}=\left\{\begin{array}{l}
{[2,2,2,3]_{12},[2,2,2,4]_{8},[2,2,2,6]_{6},[2,2,3,3]_{6},} \\
{[2,2,4,4]_{4},[3,3,3,3]_{3}}
\end{array}\right\} \\
& \mathcal{N}_{5}=\left\{[2,2,2,2,2]_{4}\right\}, \quad \mathcal{N}_{6}=\left\{[2,2,2,2,2,2]_{2}\right\} .
\end{aligned}
$$

Proof. Suppose that $\left[m_{1}, \ldots, m_{r}\right]$ is a tuple of natural numbers in $\mathcal{N}_{r}$. From condition (iv) we get

$$
\sum_{i=1}^{r}\left(1-\frac{1}{m_{i}}\right) \leq 2+\frac{2}{m_{r}} \leq 3 .
$$

Using $2 \leq m_{i}$ for $i=1, \ldots, r$ we obtain $r \leq 6$ and $r=6$ if and only if $m_{i}=2$ for all $i$. In particular, $\mathcal{N}_{r}$ is empty for $r \geq 7$ and $\mathcal{N}_{6}$ has only one element as stated.

Let us treat the case $r=3$ next. In this case we have $m_{2} \geq 3$ since otherwise $\Theta\left(\left[m_{1}, m_{2}, m_{3}\right]\right)$ is negative which contradicts condition (ii). An application of (12) using $m_{1} \geq 2$ and $m_{2} \geq 3$ gives $m_{3} \leq 18$. By a quick computer search through the remaining tuples (or just by hand) we obtain the finite set $\mathcal{N}_{3}$.

In the cases $r=4,5$ we infer from (12) that

$$
\sum_{i=1}^{r-2}\left(1-\frac{1}{m_{i}}\right) \leq \frac{1}{m_{r-1}}+\frac{3}{m_{r}},
$$

whence $\frac{r-2}{2} \leq \frac{4}{m_{r-1}}$ and $\frac{r-3}{2} \leq \frac{3}{m_{r}}$. These inequalities imply $m_{r-1} \leq \frac{8}{r-2} \leq 4$ and $m_{r} \leq \frac{6}{r-3} \leq 6$. The remaining tuples can again be quickly searched by computer or by hand to obtain the above lists for $\mathcal{N}_{4}, \mathcal{N}_{5}$.

Q.E.D. 
In the following we define properties of a tuple of natural numbers which are satisfied by the tuple of orders of the spherical system of generators occurring in the mixed case.

Definition 1.5. I) Given $A=\left[m_{1}, \ldots, m_{r}\right] \in \mathbb{N}^{r}$ define

$$
\beta(A):=\frac{4}{\Theta(A)}=\frac{4}{-2+\sum_{i=1}^{r}\left(1-\frac{1}{m_{i}}\right)}
$$

and use the notation $\left[m_{1}, \ldots, m_{r}\right]_{\beta(A)}$.

II) For $r \in \mathbb{N}$ with $r \geq 3$ let $\mathcal{M}_{r}$ be the set of $r$-tuples $\left[m_{1}, \ldots, m_{r}\right] \in \mathbb{N}^{r}$ which satisfy:

(i): $2 \leq m_{1} \leq \ldots \leq m_{r}$,

(ii): $\Theta\left(\left[m_{1}, \ldots, m_{r}\right]\right)>0$,

(iii): $\beta\left(\left[m_{1}, \ldots, m_{r}\right]\right) \in \mathbb{N}$,

(iv): $\beta\left(\left[m_{1}, \ldots, m_{r}\right]\right)$ is even,

(v): $m_{i}$ divides $\beta\left(\left[m_{1}, \ldots, m_{r}\right]\right)$ for all $1 \leq i \leq r$.

Define further $\mathcal{M}:=\cup_{i=3}^{\infty} \mathcal{M}_{i}$.

We shall now give a simple classification result for the tuples in $\mathcal{M}$.

Proposition 1.6. We have $\mathcal{M}_{r}=\emptyset$ for $r \geq 9$ or $r=7$ and

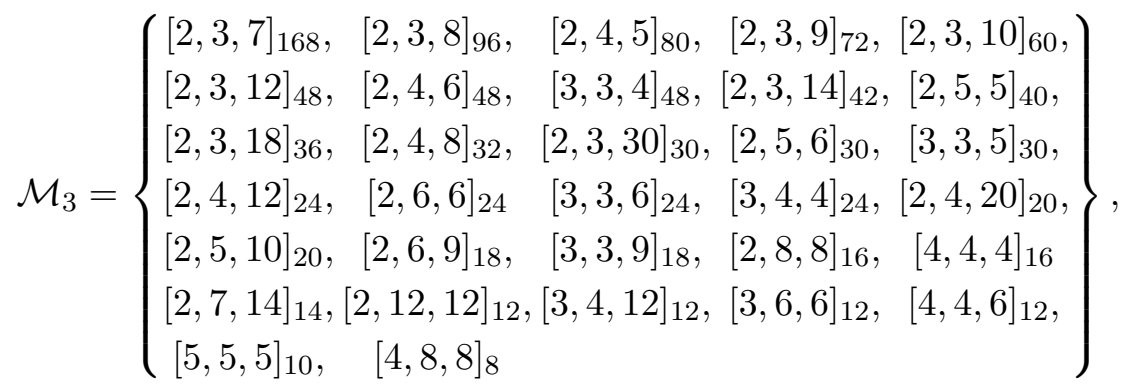

$$
\begin{aligned}
& \mathcal{M}_{4}=\left\{\begin{array}{cl}
{[2,2,2,3]_{24},} & {[2,2,2,4]_{16},[2,2,2,6]_{12},[2,2,3,3]_{12},} \\
{[2,2,2,10]_{10},} & {[2,2,4,4]_{8},[2,2,6,6]_{6},[3,3,3,3]_{6},} \\
{[2,3,3,6]_{6},} & {[4,4,4,4]_{4}}
\end{array}\right\}, \\
& \mathcal{M}_{5}=\left\{[2,2,2,2,2]_{8},[2,2,2,2,3]_{6},[2,2,2,4,4]_{4}\right\}, \\
& \mathcal{M}_{6}=\left\{[2,2,2,2,2,2]_{4}\right\}, \quad \mathcal{M}_{8}=\left\{[2,2,2,2,2,2,2,2]_{2}\right\}
\end{aligned}
$$


We skip the proof since it is similar to that of Proposition 1.4.

\section{BASiCs on SuRfaCes isogenous to a PRODUCT}

Throughout this section we assume that $S$ is a surface of general type. We recall first the notion of surfaces isogenous to a product of curves. By Proposition 3.11 of [Cat00] the following two properties for a surface of general type are equivalent.

Definition 2.1. A surface $S$ of general type is said to be isogenous to a product if and only if one of the following two equivalent conditions is satisfied.

- $S$ admits a finite unramified covering which is isomorphic to a product of curves (of genera at least two),

- $S$ is a quotient $S:=\left(C_{1} \times C_{2}\right) / G$, where the $C_{i}$ 's are curves of genus at least two, and $G$ is a finite group acting freely on $C_{1} \times C_{2}$.

It is shown in [Cat00] that every such surface isogenous to a product has a unique minimal realization $S:=\left(C_{1} \times C_{2}\right) / G$ (i.e., the genera $g\left(C_{1}\right), g\left(C_{2}\right)$ of the two curves $C_{1}, C_{2}$ are minimal).

It can further be shown (see [Cat00]) that the action of $G$ on $C_{1} \times C_{2}$ in the second condition of the above definition respects the product decomposition, i.e., the elements of $G$ either interchange the factors or act independently on both factors.

Definition 2.2. Let $S$ be a surface isogenous to a product with minimal realization $S=\left(C_{1} \times C_{2}\right) / G$. We say that $S$ is a mixed case if the action of $G$ exchanges the two factors (and then $C_{1}, C_{2}$ are isomorphic), and an unmixed case if $G$ acts via a diagonal action.

We shall associate to a surface $S$ now certain algebraic data. This approach is taken from [Cat00] where a much more detailed discussion can be found. We first take a minimal realization of $S$ as $S=\left(C_{1} \times C_{2}\right) / G$ and define

$$
G(S):=G \text {. }
$$

Suppose we are in the unmixed case. Then $q(S)=0$ implies that $C_{1} / G(S)=$ $C_{2} / G(S)=\mathbb{P}^{1}$, i.e., we have two ramified Galois coverings

$$
C_{1} \rightarrow \mathbb{P}^{1}, \quad C_{2} \rightarrow \mathbb{P}^{1}
$$


with Galois group $G$ (see [Mir], section 4 for explanations). Let $\left\{P_{1}, \ldots, P_{r}\right\} \subset \mathbb{P}^{1}$ be the set of branch points of the first covering. Choose a base point $P$ in $\mathbb{P}^{1}$ distinct from them. Choose a geometric basis $\gamma_{1}, \ldots \gamma_{r}$ of $\pi_{1}\left(\mathbb{P}^{1}-\left\{P_{1}, \ldots P_{r}\right\}\right)$ ( $\gamma_{i}$ is a simple counterclockwise loop around $P_{i}$, and they follow each other by counterclockwise ordering around the base point). Notice that $\gamma_{1} \cdot \ldots \cdot \gamma_{r}=1$. Choose a monodromy representation, i.e., a surjective homomorphism

$$
\psi: \pi_{1}\left(\mathbb{P}^{1}-\left\{P_{1}, \ldots P_{n}\right\}\right) \rightarrow G .
$$

Notice that only the kernel of $\psi$ is uniquely determined by the covering. Then the elements $\psi\left(\gamma_{1}\right), \ldots, \psi\left(\gamma_{r}\right)$ form a spherical system of generators of $G$.

Now, the mapping class group of the sphere $\pi_{0}\left(\operatorname{Diff}\left(\mathbb{P}^{1}-\left\{P_{1}, \ldots P_{n}\right\}\right)\right)$, which is a quotient of the braid group $\mathbf{B}_{n}$, operates on such homomorphisms, and their orbits are called Hurwitz equivalence classes of spherical systems of generators. This action is the one which was already described in the previous section. We use this action in order to assume without loss of generality that $T_{1}(S):=\left[\psi\left(\gamma_{1}\right), \ldots, \psi\left(\gamma_{r}\right)\right]$ is an ordered spherical system of generators.

We apply the same principle to the second covering and obtain another ordered spherical system of generators $T_{2}(S)$ of $G$.

Since the action of $G$ on $C_{1} \times C_{2}$ is free we have $\Sigma\left(T_{1}(S)\right) \cap \Sigma\left(T_{2}(S)\right)=\{1\}$, i.e., the two systems are disjoint.

Let $S$ be a surface isogenous to a product, of unmixed type and with $q(S)=0$. Then we have attached to $S$ its finite group $G=G(S)$ (up to isomorphism) and a pair $\mathcal{T}(S)=\left(T_{1}(S), T_{2}(S)\right) \in \mathcal{B}\left(G ; A_{1}(S), A_{2}(S)\right)$ of an uniquely defined ordered type $\left(A_{1}(S), A_{2}(S)\right)$.

We show now that the tuples $T_{1}(S), T_{2}(S)$ attached to a surface $S$ isogenous to a product, of unmixed type and with $p_{g}(S)=0$ satisfy the properties of section 1.2 , i.e., that they are contained in $\mathcal{N}$.

Proposition 2.3. Let $S$ be a surface isogenous to a product, of unmixed type and with $p_{g}(S)=0$. Let $A_{1}(S)=\left[m_{1}, \ldots, m_{r}\right], A_{2}(S)=\left[n_{1}, \ldots, n_{s}\right]$ be the two ordered types attached to $S$ as above. We have

- $\Theta\left(A_{1}(S)\right), \Theta\left(A_{2}(S)\right)>0$,

- $m_{i}$ divides $\frac{2}{\Theta\left(A_{1}(S)\right)}$ for all $1 \leq i \leq r, n_{s}$ divides $\frac{2}{\Theta\left(A_{2}(S)\right)}$ for all $1 \leq i \leq s$, 
- $\frac{2}{\Theta\left(A_{1}(S)\right)}, \frac{2}{\Theta\left(A_{2}(S)\right)} \in \mathbb{N}$.

Proof. Since $S$ is isogenous to a product we can represent $S$ as

$$
S=\left(C_{1} \times C_{2}\right) / G(S)
$$

where $C_{1}, C_{2}$ are two smooth projective curves with genera $g\left(C_{1}\right), g\left(C_{2}\right) \geq 2$ where the finite group $G(S)$ acts without fixed points and via an action preserving the product on $C_{1} \times C_{2}$. Since $q(S)=0$ we have $C_{1} / G(S) \cong C_{2} / G(S) \cong \mathbb{P}^{1}$ and the Hurwitz formula implies

$$
\begin{aligned}
& |G|\left(-2+\sum_{j}^{r}\left(1-\frac{1}{m_{j}}\right)\right)=2\left(g\left(C_{1}\right)-1\right), \\
& |G|\left(-2+\sum_{j}^{s}\left(1-\frac{1}{n_{j}}\right)\right)=2\left(g\left(C_{2}\right)-1\right)
\end{aligned}
$$

This establishes $\Theta\left(A_{1}(S)\right), \Theta\left(A_{2}(S)\right)>0$, because $g\left(C_{1}\right), g\left(C_{2}\right) \geq 2$.

We have

$$
K_{S}^{2}=\frac{K_{C_{1} \times C_{2}}^{2}}{|G|}=\frac{8\left(g\left(C_{1}\right)-1\right)\left(g\left(C_{2}\right)-1\right)}{|G|}=8 \chi\left(\mathcal{O}_{S}\right)=8,
$$

where the last equality holds since $p_{g}=0$.

Therefore

$$
|G(S)|=\left(g\left(C_{1}\right)-1\right)\left(g\left(C_{2}\right)-1\right)
$$

and

using formulas (20), (21) we get

$$
\frac{2}{\Theta\left(A_{1}\right)}=g\left(C_{2}\right)-1, \frac{2}{\Theta\left(A_{2}\right)}=g\left(C_{1}\right)-1 \in \mathbb{N} .
$$

This establishes the third property of $A_{1}, A_{2}$.

If $T(S)=\left[g_{1}, \ldots, g_{r}\right]$ then $g_{i}$ has order $m_{i}$ and we know that it acts with a fixed point on $C_{1}$. Hence the cyclic group $\left\langle g_{i}\right\rangle$ should have no fixed points on $C_{2}$. Let $C:=C_{2} /\left\langle g_{i}\right\rangle$ be the quotient. By Hurwitz' formula we get:

$$
2 g(C)-2=\frac{2 g\left(C_{2}\right)-2}{m_{i}} .
$$

This proves the second property.

Q.E.D. 
Let $S$ be a surface isogenous to a product, of mixed type and with $q(S)=$ 0 . Then we can attach to $S$ its finite group $G=G(S)$ and a pair $\mathcal{T}(S)=$ $(H(S), T(S)) \in \mathcal{B}(G ; A)$ of an uniquely defined ordered type $A(S)$.

Here we get the following

Proposition 2.4. Let $S$ be a surface isogenous to a product, of mixed type and with $p_{g}(S)=0$. Let $A(S)=\left[m_{1}, \ldots, m_{r}\right]$ be the ordered type attached to $S$. We have

- $\Theta(A(S)) \neq 0$,

- $\beta(A(S)):=\frac{4}{\Theta(A(S))} \in \mathbb{N}$,

- $\beta(A(S))$ is even,

- $m_{i}$ divides $\frac{4}{\Theta(A(S))}$ for all $1 \leq i \leq r$.

Proof. Noting that $H(S)$ has an unmixed ramification structure of type $(A, A)$ yielding a surface with invariants $K_{S}^{2}=16=8 \chi$, the first three properties are proven in the same way as in Proposition 2.3. For the last property observe that $G$ has order $\beta(A(S))^{2}$ and has a subgroup of index 2 .

Q.E.D.

So far we have discussed the ramification structure associated to a surface isogenous to a product. There is also a way back from ramification structures to surfaces. This construction relies on the Riemann existence theorem (see [Cat00] for details). More precisely we have

Proposition 2.5. Let $G$ be a finite group. let $A_{1}=\left[m_{11}, \ldots, m_{1 r}\right]$ be an $r$-tuple and $A_{2}=\left[m_{21}, \ldots, m_{2 s}\right]$ a s-tuple of natural numbers with $2 \leq m_{11} \leq \ldots \leq m_{1 r}$ and $2 \leq m_{21} \leq \ldots \leq m_{2 s}$. Then for any ramification structure $\mathcal{T} \in \mathcal{B}\left(G ; A_{1}, A_{2}\right)$ there is a surface isogenous to a product with $G(S)=G$ and $\mathcal{T}(S)=\mathcal{T}$.

An analogous existence result holds in the mixed case also.

\section{The unmiXed CASE, Classification of the Groups}

This section is devoted to the classification of all finite groups $G$ admitting an unmixed ramification structure of type $(A, B)$ with $A, B \in \mathcal{N}$. The result is summarized in the following

Proposition 3.1. The only finite groups $G$ admitting an unmixed ramification structure of type $(A, B)$ with $A, B \in \mathcal{N}$ are those in the following table: 


\begin{tabular}{|c|c|c|c|}
\hline$G$ & $|G|$ & $A$ & $B$ \\
\hline $\mathfrak{A}_{5}$ & 60 & {$[2,5,5]_{20}$} & {$[3,3,3,3]_{3}$} \\
$\mathfrak{A}_{5}$ & 60 & {$[5,5,5]_{5}$} & {$[2,2,2,3]_{12}$} \\
$\mathfrak{A}_{5}$ & 60 & {$[3,3,5]_{15}$} & {$[2,2,2,2]_{4}$} \\
$\mathfrak{S}_{4} \times \mathrm{Z}_{2}$ & 48 & {$[2,4,6]_{24}$} & {$[2,2,2,2,2,2]_{2}$} \\
$G(32)$ & 32 & {$[2,2,4,4]_{4}$} & {$[2,2,2,4]_{8}$} \\
$\mathrm{Z}_{5}^{2}$ & 25 & {$[5,5,5]_{5}$} & {$[5,5,5]_{5}$} \\
$\mathfrak{S}_{4}$ & 24 & {$[3,4,4]_{12}$} & {$[2,2,2,2,2,2]_{2}$} \\
$G(16)$ & 16 & {$[2,2,4,4]_{4}$} & {$[2,2,4,4]_{4}$} \\
$\mathrm{D}_{4} \times \mathrm{Z}_{2}$ & 16 & {$[2,2,2,4]_{8}$} & {$[2,2,2,2,2,2]_{2}$} \\
$\mathrm{Z}_{2}^{4}$ & 16 & {$[2,2,2,2,2]_{4}$} & {$[2,2,2,2,2]_{4}$} \\
$\mathrm{Z}_{3}^{2}$ & 9 & {$[3,3,3,3]_{3}$} & {$[3,3,3,3]_{3}$} \\
$\mathrm{Z}_{2}^{3}$ & 8 & {$[2,2,2,2,2]_{4}$} & {$[2,2,2,2,2,2]_{2}$} \\
\hline
\end{tabular}

The proof relies heavily on the use of the MAGMA-library containing either permutational representations or polycyclic presentations of all groups of order less than 2000 with the exception of the order 1024. The relevant algorithms are described in [BEick]. We proceed as follows. We consider each type $(A, B)$ separately going through the finite list of Proposition 1.4. Assume that there is a group $G$ admitting an unmixed ramification structure of type $(A, B)$ : then $|G|=\alpha(A) \alpha(B)$. If this order is less than 2000 we just go through the MAGMAlibrary and search for groups which have a disjoint pair of systems of spherical generators of type $(A, B)$. There is a huge number of groups to check but there are methods to speed up the computation. These will be described in the next two subsections where we also exhibit the arguments for $|G|=\alpha(A) \alpha(B)>2000$. Sometimes we shall have to talk about individual groups in the MAGMA-library. Here we use the terminology of MAGMA, i.e., $\operatorname{SmallGroup}(a, b)$ denotes the group of order $a$ having number $b$ in the list.

A simple but useful observation is

Lemma 3.2. Let $\left(T_{1}, T_{2}\right)$ be a disjoint pair of spherical systems of generators of a finite group $G$. Then, for every $g \in G,\left(g T_{1} g^{-1}, T_{2}\right)$ and $\left(T_{1}, g T_{2} g^{-1}\right)$ are also disjoint pairs of spherical systems of generators of $G$.

Proof. $\Sigma\left(T_{1}\right)$ and $\Sigma\left(T_{2}\right)$ are unions of conjugacy classes. Q.E.D. 
Remark 3.3. We will often use without explicit mention the $p^{\alpha} q^{\beta}$-Theorem (of Burnside) saying that every group of order $p^{\alpha} q^{\beta}$ ( $p, q$ primes) is solvable (cf. [Bu04] and [Bu11], p.323).

3.1. The case: $A=[2,3,7]_{84}, B \in \mathcal{N}$. In this section we consider the case $A=[2,3,7]_{84}$ and prove

Proposition 3.4. There is no finite group $G$ having an unmixed ramification structure of type $(A, B)$ with $A=[2,3,7]_{84}$ and $B \in \mathcal{N}$ arbitrary.

Since the finite group $G$ satisfying the conditions of Proposition 3.4 has a system of generators of type $[2,3,7]$ it has to be a non-trivial perfect group. Recall that a group $G$ is called perfect if $G=G^{\prime}$ where $G^{\prime}$ is the commutator subgroup of $G$. Notice that every quotient group of a perfect group is again perfect. We shall often exploit

Remark 3.5. Let $G$ be a non trivial finite perfect group, then $G$ has a non abelian simple group $Q$ as quotient.

The perfect groups of order less than one million are described in [HoPle], section 5.4. From this table it also can be read off that the only non abelian simple groups whose order divides 2016, 3024, 4032, 7056 are $\operatorname{PSL}\left(2, \mathbb{F}_{7}\right)$ and $\operatorname{PSL}\left(2, \mathbb{F}_{8}\right)$. This fact can be seen by applying the command "SimpleGroupsWithOrderDividing" of MAGMA to the above numbers.

Now we shall run through all $B \in \mathcal{N}$ and explain the computations that are performed.

3.1.1. $A=[2,3,7]_{84}, B \in \mathcal{N}, \alpha(B) \leq 20$. The necessary computations can be speeded up enormously by first noting (see [HoPle], section 5.4) that the only perfect groups of order $84 k$, where $k \leq 20$ is one of the numbers $\alpha(B)$ for $B \in \mathcal{N}$, are

1. $\operatorname{SmallGroup}(168,42)$ for $k=2$, 2 . $\operatorname{SmallGroup}(336,114)$ for $k=4$,

3. SmallGroup $(504,156)$ for $k=6, \quad$ 4. $\operatorname{SmallGroup}(1344,814)$ for $k=16$,

5. SmallGroup $(1344,1186)$ for $k=16$.

The first four of them have only one conjugacy class of elements of order 2 . 
This information can also be obtained by running through the relevant MAGMAlibraries checking the IsPerfect-predicate. We have prefered to give the MAGMAnotation for the groups instead of the notation in [HoPle].

We now may exclude the first four cases by verifying that for each $B \in \mathcal{N}$ with $\alpha(B)=2,4,6,16$ we have that 2 divides one of the $m_{i}$ 's. We are left with the case $B=[2,4,8]_{16}\left(\right.$ since $\alpha(B)=16$ iff $\left.B=[2,4,8]_{16}\right)$ and $G=$ SmallGroup $(1344,1186)$. By our knowledge of the simple groups of small order this group is an extension of $\operatorname{PSL}\left(2, \mathbb{F}_{7}\right)$ by a kernel of order 8 . It can be excluded noting that $\operatorname{PSL}\left(2, \mathbb{F}_{7}\right)$ has no spherical system of generators of types $[2,4,8]$, $[2,4,4],[2,2,4]$ or $[2,2,2]$.

3.1.2. $A=[2,3,7]_{84}, B \in \mathcal{N}_{3}, \alpha(B)=24$. Here the order of $G$ is 2016 . By Remark 3.5 there is a simple non abelian quotient $Q$ of $G$, which is isomorphic to $\operatorname{PSL}\left(2, \mathbb{F}_{7}\right)$ or to $\operatorname{PSL}\left(2, \mathbb{F}_{8}\right)$.

Suppose that $Q$ is $\operatorname{PSL}\left(2, \mathbb{F}_{7}\right)$ : then the kernel $K$ of the quotient homomorphism has order 12. Each group of order 12 has either a normal Sylow-2-subgroup or a normal Sylow-3-subgroup. This Sylow-subgroup, which we denote by $S$, is characteristic in $K$, hence normal in $G$. Suppose that $|S|=3$. Then $G / S$ is perfect and has order $2016 / 3=672$, but there are no perfect groups of this order (checked by MAGMA or [HoPle]). If $|S|=4$, then $G / S$ has order 2016/4 $=504$. The only perfect group of order 504 is $\operatorname{PSL}\left(2, \mathbb{F}_{8}\right)$ which is simple. This is a contradiction since $K / S$ is a normal subgroup of $G / S$.

Suppose that $Q$ is $\operatorname{PSL}\left(2, \mathbb{F}_{8}\right)$ : then the kernel $K$ of the quotient homomorphism has order 4 . Since $Q$ has to act trivially (by conjugation) on $K$. Let $K_{1}$ be a subgroup of order 2 in $K$. Clearly $K_{1}$ is normal in $G$ and $G / K_{1}$ is perfect of order 1008. There are no such groups.

This shows that there is no group $G$ of order 2016 with a spherical system of generators of type $[2,3,7]$.

3.1.3. $A=[2,3,7]_{84}, B \in \mathcal{N}_{3}, \alpha(B)=30$. Here the order of $G$ is 2520 and $B=[2,3,10]_{30}$. We find from [HoPle], section 4 that the only non abelian simple groups of order dividing 2520 are $\mathfrak{A}_{5}, \mathfrak{A}_{6}, \mathfrak{A}_{7}, \operatorname{PSL}\left(2, \mathbb{F}_{7}\right)$ and $\operatorname{PSL}\left(2, \mathbb{F}_{8}\right)$.

Let $Q$ be a simple quotient of $G$. The cases $Q=\mathfrak{A}_{5}$ and $Q=\mathfrak{A}_{6}$ cannot occur since these groups have no element of order 7 . 
$Q=\mathfrak{A}_{7}=G$ cannot occur since $\mathfrak{A}_{7}$ has only one conjugacy class of elements of order 2 and hence cannot have a disjoint pair of spherical generators of type $(A, B)$.

Suppose now that $Q=\operatorname{PSL}\left(2, \mathbb{F}_{7}\right)$ or $Q=\operatorname{PSL}\left(2, \mathbb{F}_{8}\right)$. These groups do not have an element of order 5 , hence $Q$ would have to be a quotient of $\mathbb{T}(2,3,2)$ which is a dihedral group. Tis is a contradiction.

3.1.4. $A=[2,3,7]_{84}, B \in \mathcal{N}_{3}, \alpha(B)=36$. Here the order of $G$ is 3024 . A non abelian simple quotient $Q$ of $G$ can only be $\operatorname{PSL}\left(2, \mathbb{F}_{7}\right)$ or $\operatorname{PSL}\left(2, \mathbb{F}_{8}\right)$.

Suppose that $Q=\operatorname{PSL}\left(2, \mathbb{F}_{7}\right)$, then the kernel $K$ of the quotient homomorphism from $G$ to $Q$ has order 18. The Sylow-3-subgroup $S$ of $K$ is normal, hence characteristic in $K$. It follows that $S$ is normal in $G$.

We find from [HoPle], section 5.4 that there is only one perfect group of order 336 , namely $\operatorname{SL}\left(2, \mathbb{F}_{7}\right)$. Hence $G / S$ is isomorphic to $\operatorname{SL}\left(2, \mathbb{F}_{7}\right)$. This is a contradiction because $G / S$ would be a quotient of $\mathbb{T}(2,3,7)$ and $\operatorname{SL}\left(2, \mathbb{F}_{7}\right)$ has only one element of order 2 which lies in its center.

Suppose that $Q=\operatorname{PSL}\left(2, \mathbb{F}_{8}\right)$. The kernel $K$ of the quotient homomorphism from $G$ to $Q$ has order 6. Its Sylow-3-subgroup $S$ is normal in $G$. The quotient group $G / S$ has order 1008 and is perfect. There is no such group.

This shows that there is no group $G$ of order 3024 with a spherical system of generators of type $[2,3,7]$.

3.1.5. $A=[2,3,7]_{84}, B \in \mathcal{N}_{3}, \alpha(B)=40$. Here the order of $G$ is 3360 and $B=[2,4,5]_{40}$. We find from [HoPle], section 5.4 that the only non abelian simple groups of order dividing 3360 are $\mathfrak{A}_{5}$, and $\operatorname{PSL}\left(2, \mathbb{F}_{7}\right)$. Any minimal non abelian simple quotient $Q$ of $G$ has to be one of these two groups. Using the fact that $\mathfrak{A}_{5}$ has no element of order 7 and $\operatorname{PSL}\left(2, \mathbb{F}_{7}\right)$ has no element of order 5 we see that there is no group $G$ in this section.

3.1.6. $A=[2,3,7]_{84}, B \in \mathcal{N}_{3}, \alpha(B)=48$. Here the order of $G$ is 4032 and $B=[2,3,8]_{48}$. Any minimal non abelian simple quotient $Q$ of $G$ has to be one of $\operatorname{PSL}\left(2, \mathbb{F}_{7}\right)$ or $\operatorname{PSL}\left(2, \mathbb{F}_{8}\right)$. But these two groups do not have a spherical system of generators of type $[2,3,8],[2,3,4]$ or $[2,3,2]$. 
3.1.7. $A=[2,3,7]_{84}, B \in \mathcal{N}_{3}, \alpha(B)=84$. Here the order of $G$ is 7056 . Any minimal non abelian simple quotient $Q$ of $G$ has to be $\operatorname{PSL}\left(2, \mathbb{F}_{7}\right)$ or $\operatorname{PSL}\left(2, \mathbb{F}_{8}\right)$. Let $K$ be the kernel of the quotient homomorphism. This group has order 42 or 14 and it has a normal, hence characteristic Sylow-7-subgroup $S$, which has to be normal in $G$. Then $G / S$ has order 1008 and is perfect. There are no such groups.

3.2. The case: $A=[2,3,8]_{48}, B \in \mathcal{N}$. In this section we treat the case $A=$ $[2,3,8]_{48}$ and prove

Proposition 3.6. There is no finite group $G$ having an unmixed ramification structure of type $(A, B)$ with $A=[2,3,8]_{48}$ and an arbitrary $B \in \mathcal{N}$.

Let us first consider the case $B \in \mathcal{N}_{3}$. All cases except $B=[2,3,7]_{84},[2,3,8]_{48}$ or $[2,4,8]_{16}$ can be analysed quickly by MAGMA. In fact, there is no group $G$ having simultaneously a spherical system of generators of type $A$ and one of type $B$ in these cases. The computer calculation is speeded up enormously by noting that $G$ has to be either perfect or has abelianisation equal to $\mathrm{Z}_{2}$.

In the cases $B=[2,3,7]_{84},[2,3,8]_{48}$ the order of $G$ is bigger than 2000 and there are no MAGMA-libraries of groups of such order. The first case was already treated in section 3.1.6, the second is treated below. In case $B=[2,4,8]_{16}$ the number of groups (1090235) to be considered makes the computation time consuming, so we treat it by a direct argument below.

3.2.1. $A=[2,3,8]_{48}, B \in \mathcal{N}_{3}, \alpha(B)=16$. Here we have $B=[2,4,8]_{16}$. The order of the group $G$ is $768=3 \cdot 256$ and $G$ is solvable.

The group $G$ is a quotient of $\mathbb{T}(2,3,8)$ and of $\mathbb{T}(2,4,8)$. We have

$$
\mathbb{T}(2,3,8)^{\mathrm{ab}}=\mathrm{Z}_{2}, \quad \mathbb{T}(2,3,8)^{\prime} \cong \mathbb{T}(3,3,4) .
$$

The abelianisation of $\mathbb{T}(3,3,4)$ is $\mathrm{Z}_{3}$. These facts imply that $G^{\mathrm{ab}}$ is $\mathrm{Z}_{2}$ and $\left(G^{\prime}\right)^{\mathrm{ab}}$ is $\mathrm{Z}_{3}$, since they are both not perfect.

The triangle group $\mathbb{T}(2,4,8)$ has exactly 3 subgroups of index 2 . They are isomorphic to $\mathbb{T}(2,8,8), \mathbb{T}(4,4,4)$ and $\mathbb{T}(2,2,2,4)$ and each of them has a 2group as abelianisation. This implies that the abelianisation of $G^{\prime}$ has to be a 2 -group which is a contradiction. 
The subgroups of finite index in finitely presented groups (like $\mathbb{T}(2,4,8)$ ) can quickly be analysed by the "generators and relations" programs of MAGMA to obtain results like those just used (alternatively one can use geometric branching arguments, cf. Lemma 2.3 of [Cat03]).

3.2.2. $A=[2,3,8]_{48}, B \in \mathcal{N}_{3}, \alpha(B)=48$. In this case we have $B=[2,3,8]_{48}$ and $|G|=2304$. Every group of this order is solvable.

We again use (22) to find that $G$ has to have a subgroup of index 2 with abelianisation $\mathrm{Z}_{3}$. The Smallgroups-library of MAGMA contains all groups of order 1152. It can be quickly checked that there is no group of order 1152 with abelianisation equal to $\mathrm{Z}_{3}$.

3.2.3. $A=[2,3,8]_{48}, B \in \mathcal{N}_{4}, \mathcal{N}_{5}, \mathcal{N}_{6}$. All these pairs of types $(A, B)$ can be quickly searched by computer to find

Lemma 3.1. There is no finite group $G$ having a pair of sytems of generators of types $(A, B)$ with $A=[2,3,8]_{48}, B \in \mathcal{N}_{4}, \mathcal{N}_{5}, \mathcal{N}_{6}$ with exception of SmallGroup $(96,64)$ which has non disjoint pairs of spherical systems of generators of type $(A, B)$ with $B=[2,2,2,2,2,2]_{2}$.

3.3. The case: $A, B \in \mathcal{N}_{3}, \alpha(A), \alpha(B) \leq 40$. All these pairs of types $(A, B)$ can be quickly searched by computer to find

Lemma 3.2. There is no finite group $G$ having a disjoint pair of spherical sytems of generators of types $(A, B)$ with $A, B \in \mathcal{N}_{3}$ and $\alpha(A), \alpha(B) \leq 40$ except for $G=\mathrm{Z}_{5}^{2}$ which admits such a system with $A, B=[5,5,5]_{5}$.

3.4. The case: $A \in \mathcal{N}_{3}, \alpha(A) \neq 48,84, B \in \mathcal{N}_{4}, \mathcal{N}_{5}, \mathcal{N}_{6}$. All these pairs of types $(A, B)$ can be quickly searched by computer to find as only groups $G$ with an unmixed ramification structure the following cases

- $A=[2,5,5]_{20}, B=[3,3,3,3]_{3}, G=\operatorname{SmallGroup}(60,5)$,

- $A=[5,5,5]_{5}, B=[2,2,2,3]_{12}, G=\operatorname{SmallGroup}(60,5)$,

- $A=[3,3,5]_{15}, B=[2,2,2,2,2]_{4}, G=\operatorname{SmallGroup}(60,5)$,

- $A=[2,4,6]_{24}, B=[2,2,2,2,2,2]_{2}, G=\operatorname{SmallGroup}(48,48)$,

- $A=[3,4,4]_{12}, B=[2,2,2,2,2,2]_{2}, G=\operatorname{SmallGroup}(24,12)$. 
From the description of the groups given by MAGMA it is easy to see that $\operatorname{SmallGroup}(60,5)$ is the alternating group $\mathfrak{A}_{5}, \operatorname{SmallGroup}(48,48)$ is $\mathfrak{S}_{4} \times Z_{2}$ and $\operatorname{SmallGroup}(24,12)$ is $\mathfrak{S}_{4}$.

3.5. The case: $A, B \in \mathcal{N}_{4}, \mathcal{N}_{5}, \mathcal{N}_{6}$. All these pairs of types $(A, B)$ can be quickly searched by computer to find as only groups $G$ with an unmixed ramification structure the following cases

- $A=[2,2,2,4]_{8}, B=[2,2,4,4]_{4}, G=\operatorname{SmallGroup}(32,27)$,

- $A=[2,2,4,4]_{4}, B=[2,2,4,4]_{4}, G=\operatorname{SmallGroup}(16,3)$,

- $A=[2,2,2,4]_{8}, B=[2,2,2,2,2,2]_{2}, G=\operatorname{SmallGroup}(16,11)$,

- $A=[2,2,2,2,2]_{4}, B=[2,2,2,2,2]_{4}, G=\operatorname{SmallGroup}(16,14)$,

- $A=[3,3,3,3]_{3}, B=[3,3,3,3]_{3}, G=\operatorname{SmallGroup}(9,2)$,

- $A=[2,2,2,2,2]_{4}, B=[2,2,2,2,2,2]_{2}, G=\operatorname{SmallGroup}(8,5)$.

The group $\operatorname{SmallGroup}(8,5)$ is $Z_{2}^{3}$, $\operatorname{SmallGroup}(9,2)$ is $Z_{3}^{2}$, $\operatorname{SmallGroup}(16,14)$ is $\mathrm{Z}_{2}^{4}$ and SmallGroup $(16,11)$ is $\mathrm{D}_{4} \times \mathrm{Z}_{2}$ where $\mathrm{D}_{4}$ stands for the dihedral group of order 8. A finite polycyclic presentation of $\operatorname{SmallGroup}(16,3)$ is

$$
G(16):=\operatorname{SmallGroup}(16,3)=\left\langle g_{1}, g_{2}, g_{3}, g_{4} \mid g_{1}^{2}=g_{4}, g_{2}^{g_{1}}=g_{2} g_{3}\right\rangle .
$$

Remark 3.7. The convention here is that the squares of all generators $g_{1}, \ldots, g_{4}$ which are not mentioned in the presentation are equal to 1 . If $h_{1}, h_{2}$ are elements of the group $G$ then we use the notation $h_{1}^{h_{2}}:=h_{2}^{-1} h_{1} h_{2}$. All conjugates $g_{i}^{g_{j}}$ amongst the generators which are not mentioned are equal to $g_{i}$, i.e., $g_{i}$ and $g_{j}$ commute in this case.

A finite polycyclic presentation of $G(32):=\operatorname{SmallGroup}(32,27)$ is

$$
G(32)=\left\langle g_{1}, g_{2}, g_{3}, g_{4}, g_{5} \quad \mid \quad g_{2}^{g_{1}}=g_{2} g_{4}, g_{3}^{g_{1}}=g_{3} g_{5}\right\rangle .
$$

\section{The miXed Case, Classification of the groups}

This section contains the classification of all finite groups $G$ which admit a mixed ramification structure of type $A \in \mathcal{M}$. In fact, there are only two such groups which are described in detail in section 4.2. We show

Proposition 4.1. There are two finite groups which admit a mixed ramification structure of type $A \in \mathcal{M}$. They both have order 256 and admit a structure of type $[4,4,4]_{16} \in \mathcal{M}_{3}$. 
The proof relies again heavily on the use of the MAGMA-library containing all groups of low order. In order to avoid an excess of computations we first consider each type $A=\left[m_{1}, \ldots, m_{r}\right] \in \mathcal{M}_{r}(r \in \mathbb{N})$ seperately going through the finite list of Proposition 1.6, trying in a first round to exclude as many cases as possible by some criteria, which are computationally cheap to verify.

If these are satisfied and if we have we have access to the groups of order $\beta(A)^{2} / 2$ through a MAGMA-library we check for each of these groups $H$

- does $H$ admit a spherical system of generators of type $A$ ?

- does $H$ admit a disjoint pair of spherical systems of generators of type $(A, A)$ ?

In fact, only very few groups $H$ survive the first test and for them the second criterion, though computationally expensive, can be carried out. We are left with a small list of groups $H$ admitting a disjoint pair of spherical systems of generators of type $(A, A)$, i.e., an unmixed ramification structure. Fortunately such groups $H$ only appear when the order $\beta(A)^{2}$ is small enough to have access to all groups $G$ of this order. We then go through all these groups $G$ and list their subgroups of index 2 isomorphic to one of the groups $H$. We then check whether the compatibility conditions for a mixed ramification structure of type $A$ (see section 1.1) could be satisfied.

If the order $\beta(A)^{2} / 2$ is too big to use a MAGMA-library we analyse the subgroups of low index in the polygonal group $\mathbb{T}\left(m_{1}, \ldots, m_{r}\right)$ (which often happen to be isomorphic to polygonal groups). We always can show that $H$ would then have a subgroup of low index which is a quotient of another polygonal group. Using this descent procedure, sometimes repeatedly, always brought us into a region of orders accessible to MAGMA-libraries.

Remark 4.2. Proposition (4.4) of [BCG05] contains a misprint (the order of $G$ was mistakenly confused with the order of $H$ ). The correct statement is: no group of order $<256$ admits a mixed Beauville structure, i.e., a mixed ramification structure of length 3 .

4.1. $A \in \mathcal{M}_{3}, \beta(A) \neq 16$. In this section we treat the cases $A \in \mathcal{M}_{3}, \beta(A) \neq 16$. In each case we indicate a sequence of MAGMA-computations showing that there is no finite group $G$ admitting a mixed ramification structure of such type. 
4.1.1. $A \in \mathcal{M}_{3}, \beta(A)=8,10,12,14,18,20,30,36,42,60$. In these cases the order of $H$ is either small or divisible only by a power of 2 with exponent $\leq 3$ and all relevant groups $H$ can be quickly inspected by MAGMA. We find

Lemma 4.1. Let $k$ be one of the numbers 8, 10, 12, 14, 18, 20, 30, 36, 42, 60 and $H$ a group of order $k^{2} / 2$. Then $H$ does not have a disjoint pair of spherical systems of generators of type $(A, A)$ with $\beta(A)=k$.

For $k=10,14,60$ there is even no group of order $k^{2} / 2$ having a system of generators of type $A$ with $\beta(A)=k$.

4.1.2. $A \in \mathcal{M}_{3}, \beta(A)=24$. The group $G$ has order $|G|=576=32 \cdot 27$, hence is solvable. Since the order of the subgroup $H$ is still low we may quickly infer using MAGMA

Lemma 4.2. No group $H$ of order 288 has a disjoint pair of spherical systems of generators of type $(A, A)$ with $A \in \mathcal{M}_{3}$ and $\beta(A)=24$.

4.1.3. $A \in \mathcal{M}_{3}, \beta(A)=32$. Here $A=[2,4,8]_{32}$. The group $G$ has order $|G|=$ 1024 , it is a 2-group. Its subgroup $H$ has order 512 and has a spherical system of generators of type $[2,4,8]$. A computation (of about 6 hours) using the MAGMAlibrary of groups of order 512 reveals

Lemma 4.3. There are 10494213 groups of order 512. Eight of these groups (SmallGroup(512, v) for $v=409,1818,1822,1832,1838,1854,1862,2023$ ) have a system of generators of type $[2,4,8]$.

The analysis of so many groups is made feasible by first selecting those groups of order 512 whose abelianisation is a quotient of the abelianisation of $\mathbb{T}(2,4,8)$ which is isomorphic to $\mathrm{Z}_{2} \times \mathrm{Z}_{4}$.

Now a quick computation shows

Lemma 4.4. None of the groups of order 512 admitting a spherical system of generators of type $[2,4,8]$ has a disjoint pair of spherical systems of generators of type $([2,4,8],[2,4,8])$. 
4.1.4. $A \in \mathcal{M}_{3}, \beta(A)=40$. Here $A=[2,5,5]_{40}$. The group $G$ has order $|G|=$ 1600 , hence it is solvable. Its subgroup $H$ has order 800 and has a spherical system of generators of type $[2,5,5]$. We have

$$
\mathbb{T}(2,5,5)^{\mathrm{ab}}=\mathrm{Z}_{5} .
$$

Therefore the abelianisation of $H$ is $\mathrm{Z}_{5}$. But we have

Lemma 4.5. There are 1211 groups of order 800. None of them has abelianisation $\mathrm{Z}_{5}$.

4.1.5. $A \in \mathcal{M}_{3}, \beta(A)=48$. Here we have $A=[2,3,12]_{48},[2,4,6]_{48},[3,3,4]_{48}$. Going through the MAGMA-library of groups of order $1152=24 \cdot 48$ we find

Lemma 4.6. There are 157877 groups of order 1152 .

- None of them has a system of generators of type [3,3,4],

- one of them (SmallGroup $(1152,155454))$ has a system of generators of type $[2,3,12]$,

- one of them (SmallGroup(1152,157849)) has a system of generators of type $[2,4,6]$.

Treating the two groups SmallGroup $(1152, v), v=155454,157849$, which are remaining, is computationally cheap. We have

Lemma 4.7. 1) If $[a, b, c],\left[a^{\prime}, b^{\prime}, c^{\prime}\right]$ are two systems of generators of the group $H:=$ SmallGroup $(1152,155454)$ of type $[2,3,12]$ then $a, a^{\prime}$ are conjugate in $H$.

2) If $[a, b, c],\left[a^{\prime}, b^{\prime}, c^{\prime}\right]$ are systems of generators of type $[2,4,6]$ of the group $H:=$ SmallGroup $(1152,157849)$ then $a, a^{\prime}$ are conjugate in $H$.

This shows that there are no groups $H$ of order 1152 with a disjoint pair of spherical systems of generators of one of the types above.

4.1.6. $A \in \mathcal{M}_{3}, \beta(A)=72$. Here $A=[2,3,9]_{72}$. The group $G$ has order $|G|=$ 5184 , hence it is solvable. Its index 2 subgroup $H$ has order 2592 and system of generators of type $[2,3,9]$. The order of $H$ is too big to apply the computational arguments used before. To exclude this case we argue as follows.

We have

$$
\mathbb{T}(2,3,9)^{\mathrm{ab}}=\mathrm{Z}_{3}, \quad \mathbb{T}(2,3,9)^{\prime} \cong \mathbb{T}(2,2,2,3) .
$$


The commutator subgroup $H^{\prime}$ of $H$ has order 864 and we conclude from (26) that $H^{\prime}$ is a quotient of $\mathbb{T}(2,2,2,3)$. Looking through the relevant MAGMA-library we find

Lemma 4.8. Of the 4725 groups of order 864 only the two groups $H_{1}:=$ SmallGroup $(864,2225)$ and $H_{2}:=\operatorname{SmallGroup}(864,4175)$ are quotients of $\mathbb{T}(2,2,2,3)$.

We are left with the question whether $H_{1}, H_{2}$ are the commutator subgroup of a group $H$ of order 2592 which has a disjoint pair of spherical systems of generators of type $(A, A)$. We need

Lemma 4.3. Let $\tilde{H}$ be a finite group with $\tilde{H}^{\mathrm{ab}}=\mathrm{Z}_{3}$ which has a disjoint pair of spherical systems of generators $\left(\left[h_{(1,1)}, h_{(1,2)}, h_{(1,3)}\right],\left[h_{(2,1)}, h_{(2,2)}, h_{(2,3)}\right]\right)$ with $[2,3,9]=\left[\operatorname{ord}\left(h_{(1,1)}\right), \operatorname{ord}\left(h_{(1,2)}\right), \operatorname{ord}\left(h_{(1,3)}\right)\right]$ and also $[2,3,9]$ equal to $\left[\operatorname{ord}\left(h_{(2,1)}\right)\right.$, $\left.\operatorname{ord}\left(h_{(2,2)}\right), \operatorname{ord}\left(h_{(2,3)}\right)\right]$. Then

(i) $\left[h_{(i, 1)}, h_{(i, 2)} h_{(1,1)} h_{(i, 2)}^{-1}, h_{(i, 2)}^{2} h_{(i, 1)} h_{(i, 2)}^{-2}\right](i=1,2)$ are generating tuples of elements of order 2 for $\tilde{H}^{\prime}$ such that $z_{i}:=h_{(i, 1)} \cdot h_{(i, 2)} h_{(1,1)} h_{(i, 2)}^{-1} \cdot h_{(i, 2)}^{2} h_{(i, 1)} h_{(i, 2)}^{-2}$ has order 3 .

(ii) $\tilde{\Sigma}_{1} \cap \tilde{\Sigma}_{2}=\emptyset$ where for $i=1,2$

$$
\tilde{\Sigma}_{i}:=\bigcup_{h \in \tilde{H}^{\prime}} h\left\{h_{(i, 1)}, z_{i}, z_{i}^{2}\right\} h^{-1}
$$

We skip the straightforward proof.

We finish this case by

Lemma 4.9. The groups $H_{1}, H_{2}$ in Lemma 4.8 do not have generating 3-tuples with properties (i), (ii) of Lemma 4.3.

4.1.7. $A \in \mathcal{M}_{3}, \beta(A)=80$. Here $A=[2,4,5]_{80}$. The group $G$ has order $|G|=$ 6400 , hence is solvable. Its index 2 subgroup $H$ has a system of generators of type $[2,4,5]$. We have

$$
\mathbb{T}(2,4,5)^{\mathrm{ab}}=\mathrm{Z}_{2}, \quad \mathbb{T}(2,4,5)^{\prime} \cong \mathbb{T}(2,5,5) .
$$

This implies that there must be a subgroup $H_{1}$ of index 2 in $H$ which is a quotient of $\mathbb{T}(2,5,5)$. $H_{1}$ has order 1600 and is solvable, hence it has abelianisation $Z_{5}$. An inspection of the relevant MAGMA-library shows

Lemma 4.10. There are 10281 groups of order 1600. None of them has abelianisation $\mathrm{Z}_{5}$. 
4.1.8. $A \in \mathcal{M}_{3}, \beta(A)=96$. Here $A=[2,3,8]_{96}$ and $G$ has order $|G|=96^{2}=$ 9216, hence is solvable. Its subgroup $H$ has a system of generators of type $[2,3,8]$.

We have

$$
\mathbb{T}(2,3,8)^{\mathrm{ab}}=\mathrm{Z}_{2}, \quad \mathbb{T}(2,3,8)^{\prime} \cong \mathbb{T}(3,3,4) .
$$

This implies that there must be a subgroup $H_{1}$ of index 2 in $H$ which is a quotient of $\mathbb{T}(3,3,4)$. We further have

$$
\mathbb{T}(3,3,4)^{\mathrm{ab}}=\mathrm{Z}_{3}, \quad \mathbb{T}(3,3,4)^{\prime} \cong \mathbb{T}(4,4,4) .
$$

This in turn implies ( $H$ is solvable) that there is a subgroup $H_{2}$ of index 3 in $H_{1}$ which is a quotient of $\mathbb{T}(4,4,4)$. Note that $\left|H_{2}\right|=768$.

Lemma 4.11. There are 1090235 groups of order 768 . None of them is a quotient of $\mathbb{T}(4,4,4)$.

This fact can be derived by inspection of the relevant MAGMA-library. The analysis of such a hugge number of groups is made possible by first selecting those groups of order 768 which have abelianisation which is a quotient of the abelianisation of $\mathbb{T}(4,4,4)$ which is $\mathrm{Z}_{4} \times \mathrm{Z}_{4}$. There are 1651 such groups. For them it is quickly checked whether they are a quotient of $\mathbb{T}(4,4,4)$.

4.1.9. $A \in \mathcal{M}_{3}, \beta(A)=168$. Here $A=[2,3,7]_{168}$. The group $G$ has order $|G|=28224$, its subgroup $H$ has order 14112 and has a system of generators of type $[2,3,7]$.

The group $H$ is perfect, hence has a non abelian simple group as quotient. Note that the only non abelian simple groups with order dividing 14112 are $\operatorname{PSL}\left(2, \mathbb{F}_{7}\right)$ and $\operatorname{PSL}\left(2, \mathbb{F}_{8}\right)$. Let $K$ be the kernel of the quotient homomorphism. This group has order 28 or 84 and it has a normal, hence characteristic Sylow-7-subgroup $S$.

Therefore $S$ has to be normal in $G$ and $G / S$ has order 2016 and is perfect. There are no such groups.

4.2. $A \in \mathcal{M}_{3}, \beta(A)=16$. In section 1.2 we have shown that the possible tuples are $[2,6,12]_{16},[3,3,12]_{16},[3,4,6]_{16},[2,8,8]_{16}[4,4,4]_{16}$. The first three are not possible since a group of order 128 cannot contain elements of order 3 . This leaves $[2,8,8]_{16}$ and $[4,4,4]_{16}$. 
4.2.1. $A=[2,8,8]_{16}$. Going through the list of groups of order 128 we find

Lemma 4.12. There are 2328 groups of order 128 . Only 7 of them have a spherical system of generators of type $[2,8,8]$. These are $\operatorname{SmallGroup}(128, v)$ for $v=2,48,50,77,135,137,142$.

Analysing the 7 remaining groups we find

Lemma 4.13. None of the groups SmallGroup(128, $v)(v=2,48,50,77,135$, $137,142)$ has a disjoint pair of spherical systems of generators of type $(A, A)$.

4.2.2. $A=[4,4,4]_{16}$. Going through the list of groups of order 128 we find

Lemma 4.14. There are 2328 groups of order 128. Only 4 of them have a system of generators of type $[4,4,4]$. These are $\operatorname{SmallGroup}(128, v)$ for $v=$ 36, 125, 141, 144. Only SmallGroup $(128,36)$ has a disjoint pair of systems of generators of type $(A, A)$.

This leaves us with the possibility that $H \cong \operatorname{SmallGroup}(128,36)$. Going through the groups of order 256 we find

Lemma 4.15. Of the 56092 groups of order 256 only 29 contain a subgroup of index 2 isomorphic to $\operatorname{SmallGroup}(128,36)$. They are the groups $\operatorname{SmallGroup}(128, v)$ for $v=382,414,1087,1088,1089,1090,1734,1735,1736,1737,1738,2483$, 2484, 2485, 2486, 2487, 2488, 2489, 2490, 3324, 3325, 3326, 3327, 3378, 3379, $3380,3381,3678,3679$.

Analysing the 29 remaining groups we easily find

Proposition 4.4. Of the groups of order 256 exactly

(30) $\mathrm{G}(256,1):=\operatorname{SmallGroup}(256,3678), \mathrm{G}(256,2):=\operatorname{SmallGroup}(256,3679)$

admit a mixed ramification structure of type $[4,4,4]_{16}$.

Finite polycyclic resentations for the groups $\mathrm{G}(256,1)$ and $\mathrm{G}(256,2)$ are

$$
\mathrm{G}(256,1)=\left\langle\begin{array}{l|l}
g_{1}, \ldots, g_{8} & g_{1}^{2}=g_{4} g_{5} g_{6}, g_{2}^{2}=g_{4} g_{5}, \quad g_{3}^{2}=g_{4}, \\
g_{2}^{g_{1}}=g_{2} g_{4}, g_{3}^{g_{1}}=g_{3} g_{5}, \quad g_{3}^{g_{2}}=g_{3} g_{6}, \\
g_{4}^{g_{1}}=g_{4} g_{7}, g_{4}^{g_{2}}=g_{4} g_{8}, g_{5}^{g_{1}}=g_{5} g_{7} g_{8}, \\
g_{5}^{g_{2}}=g_{5} g_{8}, g_{5}^{g_{3}}=g_{5} g_{7}, g_{6}^{g_{1}}=g_{6} g_{8}, \\
g_{6}^{g_{2}}=g_{6} g_{7}, g_{6}^{g_{3}}=g_{6} g_{8}
\end{array},,\right.
$$




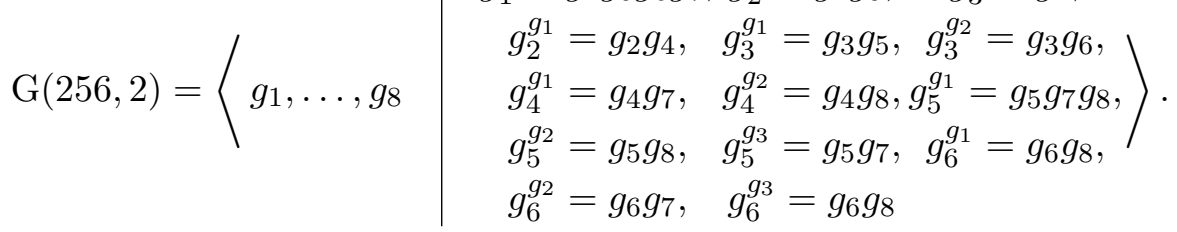

The conventions for these so called PC-presentations are explained in section 3.5.

4.3. $A \in \mathcal{M}_{4}, \mathcal{M}_{5}, \mathcal{M}_{6}, \mathcal{M}_{8}$. In this section we treat the cases $A \in \mathcal{M}_{4}, \mathcal{M}_{5}, \mathcal{M}_{6}, \mathcal{M}_{8}$. We show

Proposition 4.5. There is no finite group $G$ admitting a mixed ramification structure of type $A \in \mathcal{M}_{4}, \mathcal{M}_{5}, \mathcal{M}_{6}, \mathcal{M}_{8}$.

4.3.1. $A \in \mathcal{M}_{4}$. The order of the group $H$ is at most 288 and all relevant groups can be checked for generating systems.

\begin{tabular}{|c|c|}
\hline $\mathrm{A}$ & \\
\hline$[2,2,2,3]_{24}$ & No disjoint generating systems \\
{$[2,2,2,4]_{16}$} & No disjoint generating systems \\
{$[2,2,2,6]_{12}$} & No disjoint generating systems \\
{$[2,2,3,3]_{12}$} & No disjoint generating systems \\
{$[2,2,2,10]_{10}$} & No generating systems \\
{$[2,2,4,4]_{8}$} & SmallGroup(32,22) admits a disjoint generating systems \\
{$[2,2,6,6]_{6}$} & No disjoint generating systems \\
{$[2,3,3,6]_{6}$} & No disjoint generating systems \\
{$[3,3,3,6]_{6}$} & No generating systems \\
{$[4,4,4,4]_{4}$} & No disjoint generating systems \\
\hline
\end{tabular}

Lemma 4.16. Of the 267 groups of order 64 only 32 contain a subgroup of index 2 which is isomorphic to $\operatorname{SmallGroup}(32,22)$. None of them has a mixed ramification structure of type $[2,2,4,4]_{8}$.

4.3.2. $A \in \mathcal{M}_{5}, \mathcal{M}_{6}, \mathcal{M}_{8}$. In these cases the relevant group orders are so small that all groups $G$ can easily be inspected. There is none with a mixed ramification structure. 


\section{Moduli Spaces}

In this section we will describe completely the moduli spaces of the surfaces isogenous to a product with $p_{g}=q=0$. More precisely, let $\mathfrak{M}_{(1,8)}$ be the moduli space of minimal smooth complex projective surfaces with $\chi(S)=1$ and $K_{S}^{2}=8$. As usual $K_{S}$ denotes the canonical divisor of $S$ and $\chi(S)=1+p_{g}(S)-q(S)$ is the holomorphic Euler-Poincare' characteristic of $S$. It is nowadays wellknown (cf. [Gie77]) that $\mathfrak{M}_{(a, b)}$ is quasiprojective for all $a, b \in \mathbb{N}$. Obviously, our surfaces are contained in the moduli space $\mathfrak{M}_{(1,8)}$ and we will describe their locus there.

Let $G$ be a finite group and fix an unmixed ramification type $(A, B) \in \mathbb{N}^{r} \times \mathbb{N}^{s}$. We denote by $\mathfrak{M}_{(G ; A, B)}$ the subset of $\mathfrak{M}_{(1,8)}$ defined by isomorphism classes of surfaces isogenous to a product admitting a ramification type $(A, B)$ (or $(B, A))$.

We observe

Remark 5.1. 1) The set $\mathfrak{M}_{(G ; A, B)} \subset \mathfrak{M}_{(1,8)}$ consists of a finite number of connected components of the same dimension, which are irreducible in the Zariski topology.

2) It is clear from section 2 that the dimension $d(G ; A, B)$ of any component in $\mathfrak{M}_{(G ; A, B)}$ is precisely $\ell(A)-3+\ell(B)-3$ since we take $\ell(A)$-points in $\mathbb{P}^{1}$ modulo projective equivalence, and likewise $\ell(B)$-points in $\mathbb{P}^{1}$ modulo projective equivalence.

In order to calculate the number of components $n(G ; A, B)$ of $\mathfrak{M}_{(G ; A, B)}$ we use the following

Proposition 5.2. Let $S, S^{\prime}$ be a surfaces isogenous to a product, of unmixed type and with $q(S)=q\left(S^{\prime}\right)=0$. Then $S, S^{\prime}$ are in the same irreducible component if and only if $G(S) \cong G\left(S^{\prime}\right),\left(A_{1}(S), A_{2}(S)\right)=\left(A_{1}\left(S^{\prime}\right), A_{2}\left(S^{\prime}\right)\right)$ and $\mathcal{T}(S)$ and $\mathcal{T}\left(S^{\prime}\right)$ are in the same orbit of $\mathbf{B}_{r} \times \mathbf{B}_{s} \times \operatorname{Aut}(G)$ where $r=\ell\left(T_{1}\right), s=\ell\left(T_{2}\right)$.

For a proof we refer to [BaCa04].

By computer calculation we obtain the following table of the possible unmixed ramification structures on finite groups of type $(A, B)$ with $A, B \in \mathcal{N}$ leading to surfaces with $K^{2}=8$.

Theorem 5.3. If $S \neq \mathbb{P}^{1} \times \mathbb{P}^{1}$ is a smooth projective surface isogenous to a product of unmixed type with $p_{g}(S)=q(S)=0$ and with minimal realization 
$S \cong\left(C_{1} \times C_{2}\right) / G$ then $G$ is one of the groups in the following table and the genera of the curves $C_{1}, C_{2}$ are as listed in the table. The numbers of components $N$ in $\mathfrak{M}_{(1,8)}$ and their dimension is given in the remaining two columns.

\begin{tabular}{|c|c|c|c|c|c|}
\hline$G$ & $|G|$ & $A$ & $B$ & $n(G ; A, B)$ & $d(G ; A, B)$ \\
\hline $\mathfrak{A}_{5}$ & 60 & {$[2,5,5]_{20}$} & {$[3,3,3,3]_{3}$} & 1 & 1 \\
$\mathfrak{A}_{5}$ & 60 & {$[5,5,5]_{5}$} & {$[2,2,2,3]_{12}$} & 1 & 1 \\
$\mathfrak{A}_{5}$ & 60 & {$[3,3,5]_{15}$} & {$[2,2,2,2]_{4}$} & 1 & 1 \\
$\mathfrak{S}_{4} \times \mathrm{Z}_{2}$ & 48 & {$[2,4,6]_{24}$} & {$[2,2,2,2,2,2]_{2}$} & 1 & 3 \\
$G(32)$ & 32 & {$[2,2,4,4]_{4}$} & {$[2,2,2,4]_{8}$} & 1 & 2 \\
$\mathrm{Z}_{5}^{2}$ & 25 & {$[5,5,5]_{5}$} & {$[5,5,5]_{5}$} & 2 & 0 \\
$\mathfrak{S}_{4}$ & 24 & {$[3,4,4]_{12}$} & {$[2,2,2,2,2,2]_{2}$} & 1 & 3 \\
$G(16)$ & 16 & {$[2,2,4,4]_{4}$} & {$[2,2,4,4]_{4}$} & 1 & 2 \\
$\mathrm{D}_{4} \times \mathrm{Z}_{2}$ & 16 & {$[2,2,2,4]_{8}$} & {$[2,2,2,2,2,2]_{2}$} & 1 & 4 \\
$\mathrm{Z}_{2}^{4}$ & 16 & {$[2,2,2,2,2]_{4}$} & {$[2,2,2,2,2]_{4}$} & 1 & 4 \\
$\mathrm{Z}_{3}^{2}$ & 9 & {$[3,3,3,3]_{3}$} & {$[3,3,3,3]_{3}$} & 1 & 2 \\
$\mathrm{Z}_{2}^{3}$ & 8 & {$[2,2,2,2,2]_{4}$} & {$[2,2,2,2,2,2]_{2}$} & 1 & 5 \\
\hline
\end{tabular}

The case $G$ abelian was done in [BaCa04]. In [Pa03] four of the non abelian cases are constructed and for three of these the irreducibility of the corresponding family is proven.

We now turn to the mixed case.

Let $G$ be a finite group and fix a mixed ramification type $A$. We denote by $\mathfrak{M}_{(G ; A)}$ the subset of $\mathfrak{M}_{(1,8)}$ given by the isomorphism classes of surfaces isogenous to a product admitting a mixed ramification type $A$.

Also here we have

Remark 5.4. The set $\mathfrak{M}_{(G ; A)} \subset \mathfrak{M}_{(1,8)}$ consists of a finite number of connected components of the same dimension $d(G ; A)=\ell(A)-3$, which are irreducible in the Zariski topology.

Proposition 5.5. Let $S, S^{\prime}$ be surfaces isogenous to a product, of mixed type and with $q(S)=q\left(S^{\prime}\right)=0$. Then $S, S^{\prime}$ are in the same irreducible component if and only if $G(S) \cong G\left(S^{\prime}\right)$ and $\mathcal{T}(S)$ and $\mathcal{T}\left(S^{\prime}\right)$ are in the same orbit of $\mathbf{B}_{r} \times \operatorname{Aut}(G)$ where $r=\ell(T)$. 
Hence the number of components $n(G ; A)$ of $\mathfrak{M}_{(G ; A)}$ is precisely the number of orbits of $\mathbf{B}_{\ell(A)} \times \operatorname{Aut}(G)$ on the set $\mathcal{B}(G ; A)$.

We already know from section 4 that there are exactly the groups $\mathrm{G}(256,1)$, $\mathrm{G}(256,2)$ which have a mixed ramification structure of type $A \in \mathcal{M}$. In fact, they both have such a structure of type $A=[4,4,4]_{16}$. We shall now determine the numbers of orbits of $\mathbf{B}_{3} \times \operatorname{Aut}(\mathrm{G}(256, i)(i=1,2)$ on the set of ramification structures.

Let us begin with $G=\mathrm{G}(256,1)$. We have

Proposition 5.6. (i) The automorphism group of $\mathrm{G}(256,1)$ has 12288 elements, it acts with 3 orbits on the set of subgroups of index 2 in $\mathrm{G}(256,1)$.

(ii) Representatives for the 3 orbits are $H_{1}:=\left\langle g_{1}, g_{3}\right\rangle$ (which is a fixed point for $\operatorname{Aut}(\mathrm{G}(256,1))$ ), $H_{2}:=\left\langle g_{1}, g_{2}\right\rangle$ (which has an orbit of cardinality 3 , $H_{3}:=\left\langle g_{2}, g_{1} g_{3}\right\rangle$ (which has an orbit of cardinality 3 ).

(iii) The action of $\mathbf{B}_{3} \times \operatorname{Aut}\left(\mathrm{G}(256,1)\right.$ on $\mathcal{B}\left(\mathrm{G}(256,1) ;[4,4,4]_{16}\right)$ has 3 orbits (corresponding to the 3 orbits of $\operatorname{Aut}(\mathrm{G}(256,1))$ on the set of subgroups of index 2 in $\mathrm{G}(256,1)$.

For the group $G=\mathrm{G}(256,2)$ the picture is different, we find

Proposition 5.7. (i) The automorphism group of $\mathrm{G}(256,2)$ has 86016 elements, it acts transitively on the set of subgroups of index 2 in $\mathrm{G}(256,2)$.

(ii) The action of $\mathbf{B}_{3} \times \operatorname{Aut}(\mathrm{G}(256,2))$ on $\mathcal{B}\left(\mathrm{G}(256,2) ;[4,4,4]_{16}\right)$ is transitive.

The proof of the above two propositions is done by standard MAGMA routines.

Combining these results we find the following table of the possible mixed ramification structures on finite groups of type $A$ with $A \in \mathcal{M}$.

Theorem 5.8. If $S$ is a smooth projective surface isogenous to a product of mixed type with $p_{g}(S)=q(S)=0$ and with minimal realization $S \cong\left(C_{1} \times C_{2}\right) / G$, then $G$ is one of the groups in the following table and the genera of the curves $C_{1}, C_{2}$ are as listed in the table. The numbers $N:=n(G ; A)$ of components of $\mathfrak{M}_{(1,8)}$ and their dimension is given in the remaining two columns.

\begin{tabular}{|c|c|c|c|c|}
\hline$G$ & $|G|$ & $A$ & $n(G ; A)$ & $d(G ; A)$ \\
\hline$G=\mathrm{G}(256,1)$ & 256 & {$[4,4,4]_{16}$} & 3 & 0 \\
$G=\mathrm{G}(256,2)$ & 256 & {$[4,4,4]_{16}$} & 1 & 0 \\
\hline
\end{tabular}




\section{Concrete models}

In this section we want to give explicit descriptions of the groups and spherical systems of generators occurring in the nonabelian case (the abelian case is fully classified and described in $[\mathrm{BaCa} 04])$. Some of these nonabelian examples were already described in [BaCa04], but we thought it would be worthwhile to give a complete list.

6.1. $G=\mathfrak{A}_{5}$. An unmixed ramification structure of type $([3,3,3,3],[2,5,5])$ is given by the following tuple of elements of $\mathfrak{A}_{5}$ :

$$
([(1,2,3),(3,4,5),(4,3,2),(2,1,5)],[(2,4)(3,5),(2,1,3,4,5),(1,2,3,4,5)]) .
$$

An unmixed ramification structure of type $([5,5,5],[2,2,2,3])$ is given by the following tuple of elements of $\mathfrak{A}_{5}$ :

$([(1,2,5,3,4),(1,2,4,5,3),(1,2,3,4,5)],[(1,2)(3,4),(2,4)(3,5),(1,4)(3,5),(2,3,4)])$.

An unmixed ramification structure of type $([2,2,2,2,2],[3,3,5])$ is given by the following elements of $\mathfrak{A}_{5}$ :

$([(1,2)(3,4),(1,3)(2,4),(1,4)(2,3),(1,4)(2,5),(1,4)(2,5)],[(1,2,3),(3,4,5),(5,4,3,2,1)])$.

6.2. $G=\mathfrak{D}_{4} \times \mathrm{Z}_{2}$. We write as customary $\mathfrak{D}_{4}$ as the group generated by elements $x, y$ satisfying the relations $x^{4}=y^{2}=1, y x y=x^{-1}$.

Then there is exactly one class of unmixed ramification structures, of type

$$
([2,2,2,2,2,2],[2,2,2,4])
$$

given by the following elements of $\mathfrak{D}_{4} \times \mathrm{Z}_{2}$

$$
\left(\left[(y, 0),(y x, 1),\left(y x^{2}, 0\right),(y x, 1),\left(x^{2}, 1\right),\left(x^{2}, 1\right)\right],[(1,1),(y, 1),(x y, 0),(x, 0)]\right) .
$$

6.3. $G=\mathfrak{S}_{4}$. There is exactly one class of unmixed ramification structures, of type

$$
([2,2,2,2,2,2],[3,4,4])
$$

given by the following elements of $\mathfrak{S}_{4}$ :

$$
([(1,2),(1,2),(2,3),(2,3),(3,4),(3,4)],[(1,2,3),(1,2,3,4),(1,2,4,3)]) .
$$

Note that this generating system is contained in the ArXiv version of [BaCa04], it was not possible for technical reasons to correct the printed version in time. 
6.4. $G=\mathfrak{S}_{4} \times \mathrm{Z}_{2}$. There is exactly one class of unmixed ramification structures, of type

$$
([2,4,6],[2,2,2,2,2,2])
$$

given by the following elements of $\mathfrak{S}_{4} \times \mathrm{Z}_{2}$ :

$$
\begin{gathered}
([[(1,2), 0],[(1,2,3,4), 1],[(4,3,2), 1]], \\
[[(1,2)(3,4), 1],[(1,2), 1],[(3,4), 1],[(2,3)(1,4), 1],[(2,3), 1],[(1,4), 1]]) .
\end{gathered}
$$

6.5. $G=G(16)$. We use here the following realization of $G:=G(16)$ as a semidirect product

$$
\left(\mathrm{Z}_{4} \times \mathrm{Z}_{2}\right) \rtimes_{\Phi} \mathrm{Z}_{2}
$$

generated by $x, y, z$, with centre $C \cong \mathrm{Z}_{2} \times \mathrm{Z}_{2}$ generated by $x^{2}, y$, and such that $z x z=x y$.

There is exactly one class of unmixed ramification structures, of type

$$
([2,2,4,4],[2,2,4,4])
$$

given by the following elements of $G(16)$ :

$$
\left(\left[z, z, x, x^{-1}\right],\left[z x^{2} y, z x^{2} y, x y z,(x y z)^{-1}\right]\right) .
$$

6.6. $G=G(32), G(256,1), G(256,2)$. We construct now concrete models for the finite groups like $G(256,1)$ which make hand computations simple. We start off by giving a general construction principle for metabelian groups. A group is called metabelian if it contains an abelian normal subgroup with abelian quotient.

Let now $N, Q$ be two abelian groups written additively. Let

$$
\Phi: Q \rightarrow \operatorname{Aut}(N), \quad \Phi: q \mapsto \Phi_{q} \quad(q \in Q)
$$

a homomorphism from $Q$ to the automorphism group of $N$. Further let

$$
\Theta: Q \times Q \rightarrow N
$$

be a bilinear map. We define a multiplication on the set $N \times Q$ by setting

$$
\left(n_{1}, q_{1}\right) \cdot\left(n_{2}, q_{2}\right):=\left(n_{1}+\Phi_{q_{1}}\left(n_{2}\right)+\Theta\left(q_{1}, q_{2}\right), q_{1}+q_{2}\right)
$$

for $n_{1}, n_{2} \in N$ and $q_{1}, q_{2} \in Q$. We obtain a group structure iff

$$
\Phi_{q_{1}}\left(\Theta\left(q_{2}, q_{3}\right)\right)=\Theta\left(q_{2}, q_{3}\right)
$$


holds for all $q_{1}, q_{2}, q_{3}$. The resulting group is denoted by

$$
N \rtimes_{\Phi, \Theta} Q .
$$

There is the obvious exact sequence

$$
\langle 0\rangle \rightarrow N \rightarrow N \rtimes_{\Phi, \Theta} Q \rightarrow Q \rightarrow\langle 0\rangle
$$

hence $N \rtimes_{\Phi, \Theta} Q$ is metabelian. Conversely, every metabelian group arises in this way. If $\Theta: Q \times Q \rightarrow N$ is the zero map then $N \rtimes_{\Phi, \Theta} Q$ is a semidirect product of $N$ and $Q$ which we denote by $N \rtimes_{\Phi} Q$.

We shall now describe the models for the remaining finite groups from sections 3 and 4 .

$\mathrm{G}(32)$ : This group has nilpotency class 2 and is a semidirect product of $\mathrm{Z}_{2}^{4}$ by $\mathrm{Z}_{2}$. The homomorphism $\Phi: \mathrm{Z}_{2} \rightarrow \operatorname{Aut}\left(\mathrm{Z}_{2}^{4}\right)=\mathrm{GL}\left(4, \mathbb{F}_{2}\right)$ can be given by the single matrix (unipotent of order 2). We set

$$
\Phi_{1}:=\left(\begin{array}{llll}
1 & 0 & 0 & 0 \\
0 & 1 & 0 & 0 \\
1 & 0 & 1 & 0 \\
0 & 1 & 0 & 1
\end{array}\right)
$$

From the presentation (24) it can be seen that the resulting group $Z_{2}^{4} \rtimes_{\Phi} Z_{2}$ is isomorphic to $\mathrm{G}(32)$.

An unmixed ramification structure $\mathcal{T}=\left(T_{1}, T_{2}\right)$ of type $\left([2,2,2,4]_{8},[2,2,4,4]_{4}\right)$ on $\mathrm{Z}_{2}^{4} \rtimes_{\Phi} \mathrm{Z}_{2}$ is given by

$$
\begin{aligned}
& T_{1}=\left[\left((0,0,1,1)^{t}, 1\right),\left((1,1,1,1)^{t}, 0\right),\left((1,0,1,1)^{t}, 0\right),\left((0,1,1,1)^{t}, 1\right)\right], \\
& T_{2}=\left[\left((1,1,1,0)^{t}, 0\right),\left((1,0,0,0)^{t}, 0\right),\left((1,1,1,0)^{t}, 1\right),\left((1,0,1,0)^{t}, 1\right)\right] .
\end{aligned}
$$

G(256,1): This group has nilpotency class 3 . But fortunately for us every group with this property is metabelian. The group $G(256,1)$ is of the form $Z_{2}^{5} \rtimes_{\Phi, \Theta} Z_{2}^{3}$. We shall first describe the maps $\Phi$ and $\Theta$. 
Let $e_{1}, e_{2}, e_{3}$ be the standard basis of $\mathrm{Z}_{2}^{3}$. The homomorphism $\Phi: \mathrm{Z}_{2}^{3} \rightarrow$ $\operatorname{Aut}\left(Z_{2}^{5}\right)=\operatorname{GL}\left(5, \mathbb{F}_{2}\right)$ can be given by its values on $e_{1}, e_{2}, e_{3}$. We set

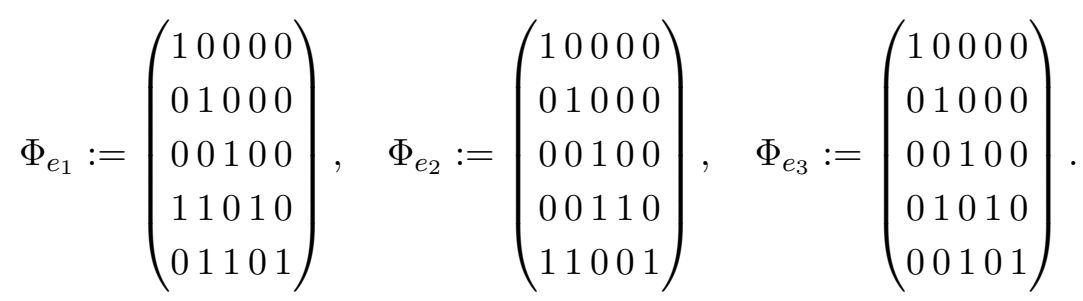

To give the bilinear map $\Theta$ we set

$$
\begin{aligned}
& \Theta\left(e_{1}, e_{1}\right):=(1,1,1,0,0)^{t}, \Theta\left(e_{2}, e_{2}\right):=(1,1,0,0,0)^{t}, \Theta\left(e_{3}, e_{3}\right):=(1,0,0,0,0)^{t}, \\
& \Theta\left(e_{2}, e_{1}\right):=(1,0,0,1,1)^{t}, \Theta\left(e_{3}, e_{1}\right):=(0,1,0,0,1)^{t}, \Theta\left(e_{3}, e_{2}\right):=(0,0,1,1,1)^{t}
\end{aligned}
$$

with the convention that the $\Theta\left(e_{i}, e_{j}\right)$ which are not mentioned are equal to 0 . From the presentation (31) it can be seen that the resulting group $Z_{2}^{5} \rtimes_{\Phi, \Theta} Z_{2}^{3}$ is isomorphic to $\mathrm{G}(256,1)$.

Here are three mixed ramification structures of type $[4,4,4]_{16}$ on $Z_{2}^{5} \rtimes_{\Phi, \theta} Z_{2}^{3}$ :

$$
\begin{gathered}
T_{1}:=\left[\left((0,0,1,0,1)^{t}, e_{3}\right),\left((1,1,0,0,0)^{t}, e_{1}\right),\left((1,0,0,1,0)^{t}, e_{1}+e_{3}\right)\right], \\
T_{2}:=\left[\left((0,0,0,0,1)^{t}, e_{2}\right),\left((1,0,0,1,0)^{t}, e_{1}+e_{2}\right),\left((0,0,1,0,1)^{t}, e_{1}\right)\right], \\
T_{3}:=\left[\left((0,0,0,1,0)^{t}, e_{2}\right),\left((1,1,0,1,0)^{t}, e_{1}+e_{2}+e_{3}\right),\left((1,0,1,1,0)^{t}, e_{1}+e_{3}\right)\right] .
\end{gathered}
$$

This is to say the three coordinates of $T_{1}, T_{2}, T_{3}$ generate a subgroup $H$ of index 2 in $G$ and the compatibility conditions of Definition 1.2 are satisfied. Moreover $T_{1}, T_{2}, T_{3}$ represent the three orbits appearing in Proposition 5.6.

$\mathrm{G}(256,2)$ : This group has nilpotency class 3 and is of the form $Z_{2}^{5} \rtimes_{\Phi, \Theta} Z_{2}^{3}$. We shall describe the maps $\Phi$ and $\Theta$.

Let $e_{1}, e_{2}, e_{3}$ be the standard basis of $\mathrm{Z}_{2}^{3}$. The homomorphism $\Phi: \mathrm{Z}_{2}^{3} \rightarrow$ $\operatorname{Aut}\left(\mathrm{Z}_{2}^{5}\right)=\mathrm{GL}\left(5, \mathbb{F}_{2}\right)$ can be given by its values on $e_{1}, e_{2}, e_{3}$. We set

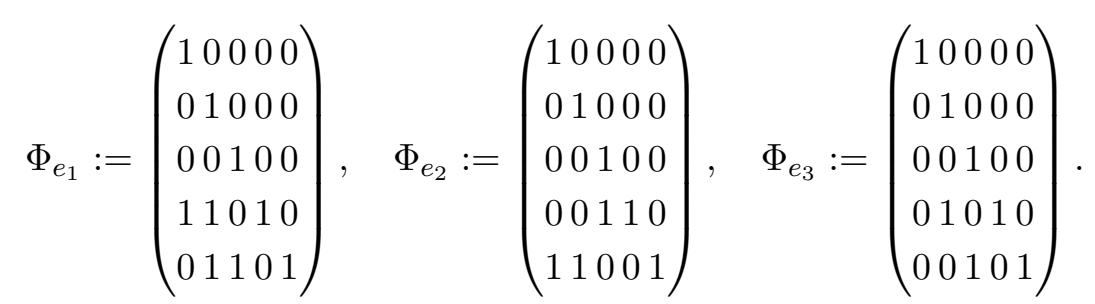

To give the bilinear map $\Theta$ we set

$$
\Theta\left(e_{1}, e_{1}\right):=(1,1,1,1,0)^{t}, \Theta\left(e_{2}, e_{2}\right):=(1,1,0,0,0)^{t}, \Theta\left(e_{3}, e_{3}\right):=(1,0,0,0,0)^{t},
$$




$$
\Theta\left(e_{2}, e_{1}\right):=(1,0,0,1,1)^{t}, \Theta\left(e_{3}, e_{1}\right):=(0,1,0,0,1)^{t}, \Theta\left(e_{3}, e_{2}\right):=(0,0,1,1,1)^{t}
$$

with the convention that the $\Theta\left(e_{i}, e_{j}\right)$ which are not mentioned are equal to 0 . From the presentation (32) it can be seen that the resulting group $Z_{2}^{5} \rtimes_{\Phi, \Theta} Z_{2}^{3}$ is isomorphic to $\mathrm{G}(256,2)$.

A mixed ramification structure of type $\left([4,4,4]_{16}\right.$ on $\mathrm{Z}_{2}^{5} \rtimes_{\Phi, \theta} \mathrm{Z}_{2}^{3}$ is given by

$$
\left[\left((0,1,0,1,0)^{t}, e_{3}\right),\left((0,0,1,0,1)^{t}, e_{2}+e_{3}\right),\left((0,0,0,0,0)^{t}, e_{2}\right)\right] .
$$

The conventions are the same as in the example $\mathrm{G}(256,1)$.

\section{REFERENCES}

[BHPV] Barth, W., Hulek, K., Peters, C., Van de Ven, A., Compact complex surfaces. Ergebnisse der Mathematik und ihrer Grenzgebiete 4, Springer-Verlag, Berlin,(2004).

[BaCa04] Bauer, I., Catanese, F.,Some new surfaces with $p_{g}=q=0$. Proceedings of the Fano Conference Torino (2004), 123-142.

[BCG05] I. Bauer, F. Catanese, F. Grunewald, Beauville surfaces without real structures, In: Geometric Methods in Algebra and Number Theory, ed. by F. Bogomolov, Y. Tschinkel, Progress in Math. 235, Birkhäuser (2005), 1-42.

[Bea78] A. Beauville, Surfaces algébriques complexes. Asterisque 54, Soc. Math. France, (1978).

[BEick] H.-U. Besche, B. Eick, The groups of order at most 1000 except 512 and 768. J. Symbolic Comput. 27, 405-413, (1999).

[Breu] T. Breuer, Characters and Automorphism Groups of Compact Riemann Surfaces. London Mathematical Society Lecture Note Series 280, Cambridge University Press, (2000).

[Bu04] Burnside, W., On groups of order $p^{a} q^{b}$. Proc. Lond. Math. Soc., p. 388 (1904).

[Bu11] Burnside, W., Theory of groups of finite order. Cambridge University Press (1911), Dover reprint (1955).

[Cam32] L. Campedelli, Sopra alcuni piani doppi notevoli con curve di diramazione del decimo ordine. Atti Acad. Naz. Lincei 15, (1932), 536-542.

[Cat00] Catanese, F., Fibred surfaces, varieties isogenous to a product and related moduli spaces. Am. J. of Math. 122,(2000), 1-44.

[Cat03] Catanese, F., Moduli spaces of surfaces and real structures. Ann. of Math. 158 , (2003), 539-554.

[Don96] S.K. Donaldson, The Seiberg-Witten Equations and 4-manifold topology. Bull. Am. Math. Soc., (N S) 33, 1, (1996) 45-70.

[Enr96] Enriques, F., Introduzione alla geometria sopra le superficie algebriche. Memorie della Societa' Italiana delle Scienze (detta "dei XL"), s.3, to. X , (1896), 1-81.

[EnrMS] Enriques, F., Memorie scelte di geometria, vol. I, II, III. Zanichelli, Bologna, (1956), 541 pp., (1959), 527 pp., (1966), 456 pp. . 
[Fre82] M. Freedman, On the topology of 4- manifolds., J. Diff. Geom. 17 (1982), 357-454.

[Gie77] Gieseker, D.: Global moduli for surfaces of general type. Invent. Math. 43, no. 3, 233-282 (1977).

[Go35] Godeaux, L., Les involutions cycliques appartenant á une surface algébrique. Actual. Sci. Ind., 270, Hermann, Paris, (1935).

[HoPle] Holt, D.F., Plesken, W., Perfect Groups. Oxford Mathematical Monographs, Clarendon Press, (1989).

[Ku] Kuga, M., FAFA Note. (1975).

[MSG] MAGMA Database of Small Groups; http://magma.maths.usyd.edu.au/magma/htmlhelp/text404.htm.

[MLP01] Mendes Lopes, M., Pardini, R., The bicanonical map of surfaces with $p_{g}=0$ and $K^{2} \geq 7$. Bull. London Math. Soc. 33 (2001), no. 3, 265-274.

[Mir] Miranda, R., Algebraic Curves and Riemann surfaces. Graduate Studies in Mathematics 5, Am. Math Soc. (1995).

[MLP03] M. Mendes Lopes, R. Pardini, The bicanonical map of surfaces with $p_{g}=0$ and $K^{2} \geq 7$. Bull. London Math. Soc. 35 (2003), no. 3, 337-343.

[Pa03] Pardini, R., The classification of double planes of general type with $K^{2}=8$ and $p_{g}=0$. J. Algebra 259 (2003), no. 1, 95-118.

[Sha78] Shavel, I. H., A class of algebraic surfaces of general type constructed from quaternion algebras. Pacific J. Math. 76, (1978), no. 1, 221-245.

[Y77] Yau, S.T., Calabi's conjecture and some new results in algebraic geometry. Proc. Nat. Acad. Sci. U.S.A. 74 (1977), no. 5, 1798-1799.

I. C. Bauer and F. Catanese

Mathematisches Institut der Universität Bayreuth

Universitätsstr. 30, 95447 Bayreuth

E-mail: Ingrid.Bauer@uni-bayreuth.de

E-mail: Fabrizio.Catanese@uni-bayreuth.de

F. Grunewald

Mathematisches Institut der Heinrich-Heine-Universität Düsseldorf

Universitätsstr.

40225 Düsseldorf

E-mail: grunewald@math.uni-duesseldorf.de 